Supporting Information: Intramolecular [2+2] and [4+2] Cycloaddition Reactions of Cinnamylamides of Ethenetricarboxylate in Sequential Processes, Shoko Yamazaki, * Hirotaka Sugiura, Shinnosuke Ohashi, Keisuke Ishizuka, Rina Saimu, Yuji Mikata, and Akiya Ogawa

III. Copies of the the 2D NOESY spectra

NOESY $\left(\mathrm{CDCl}_{3}, 400 \mathrm{MHz}\right)$

3a
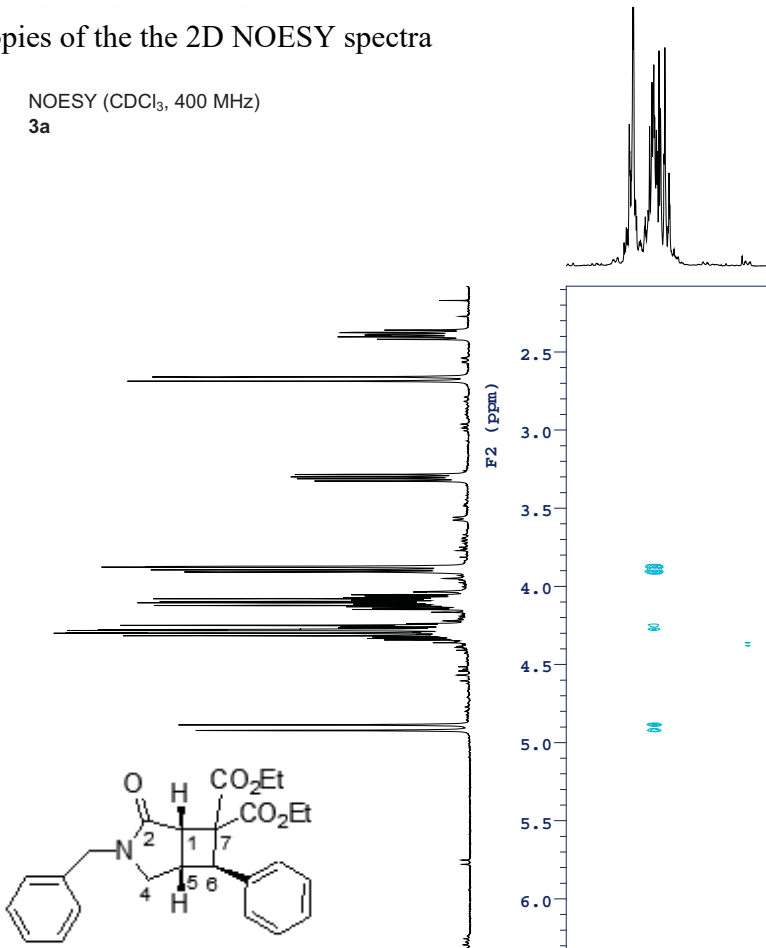

$3 a$

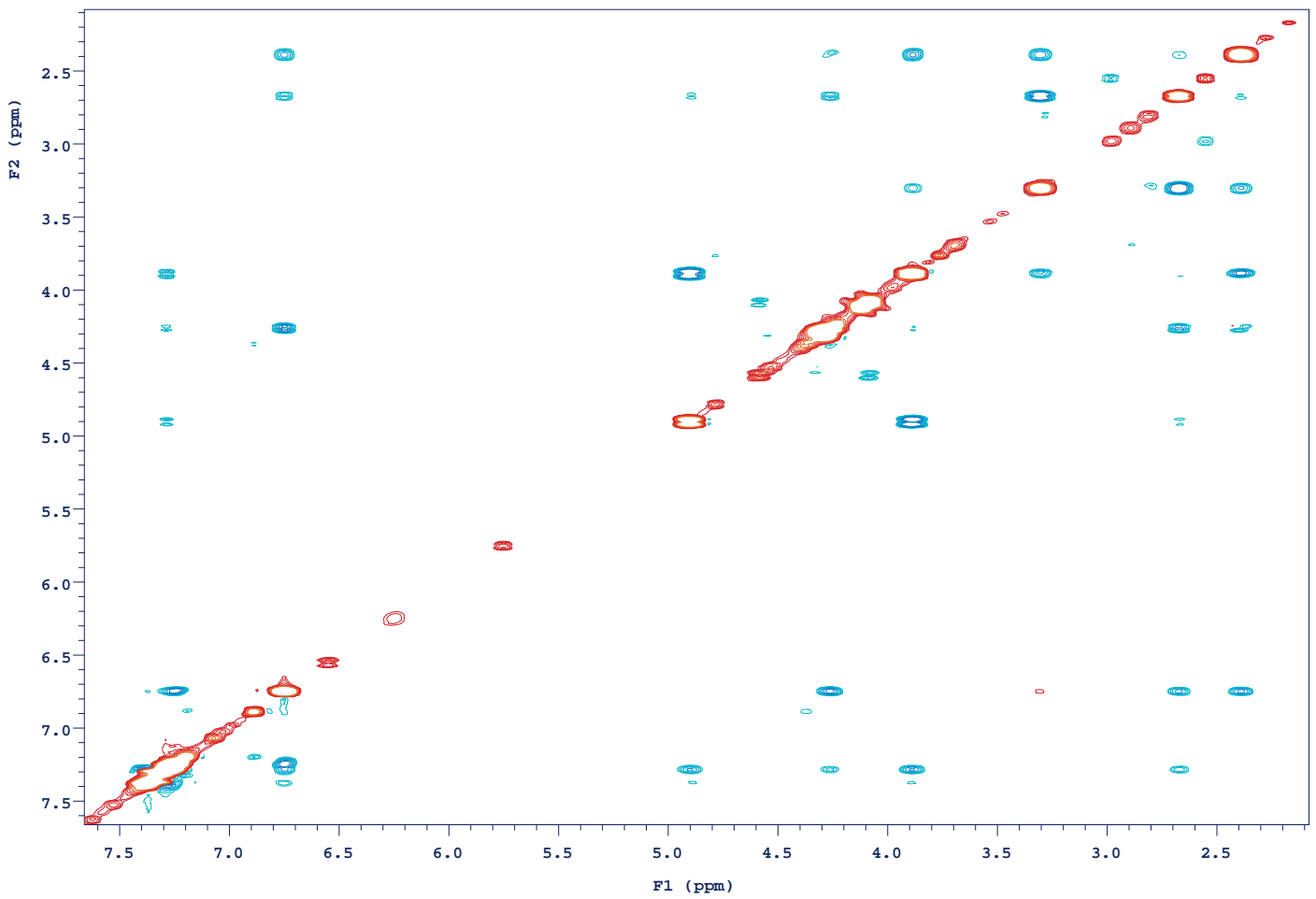

क. Agilent Technologies

NOESY $\left(\mathrm{CDCl}_{3}, 400 \mathrm{MHz}\right)$

$3 \mathrm{~b}$
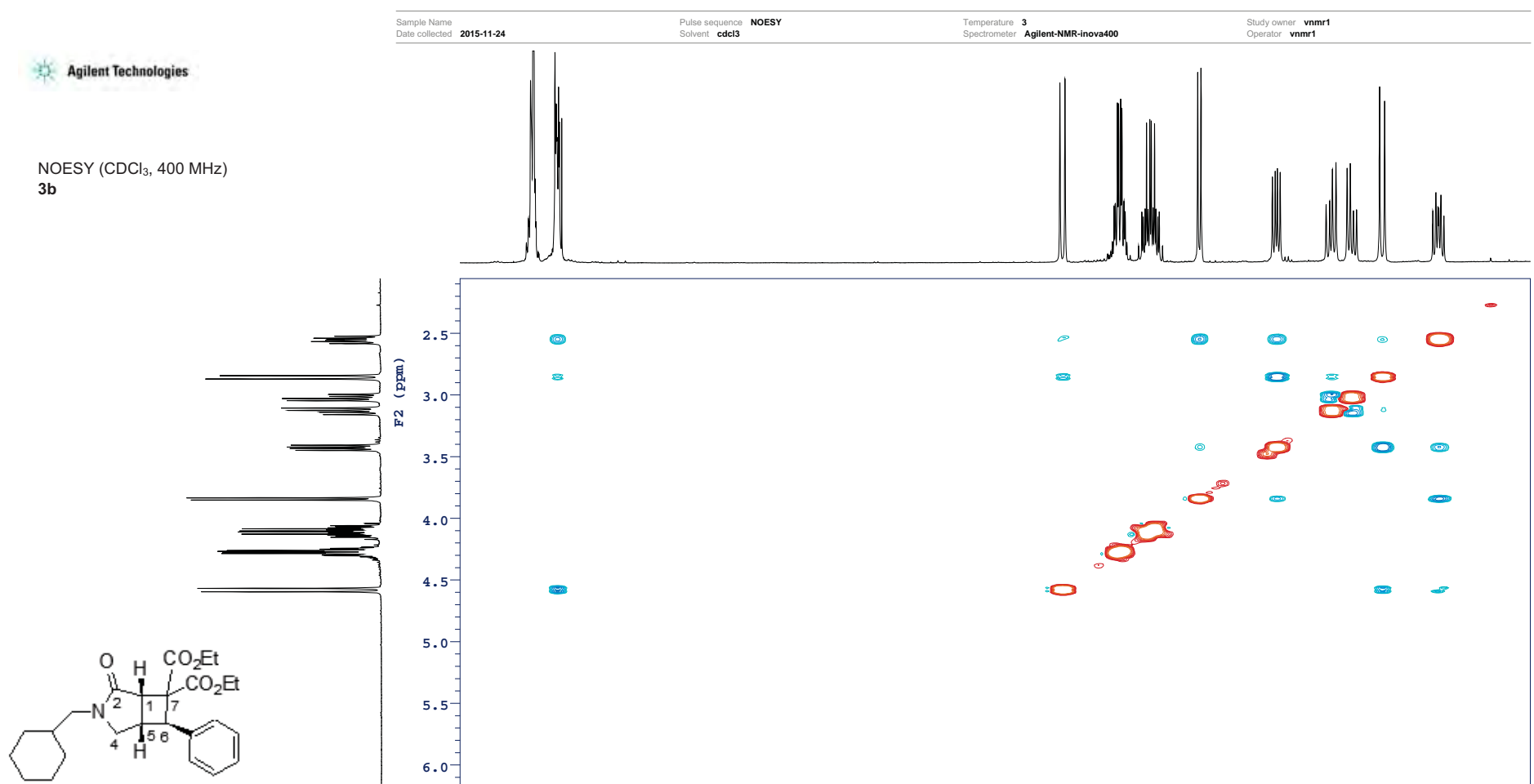

$3 \mathbf{b}$

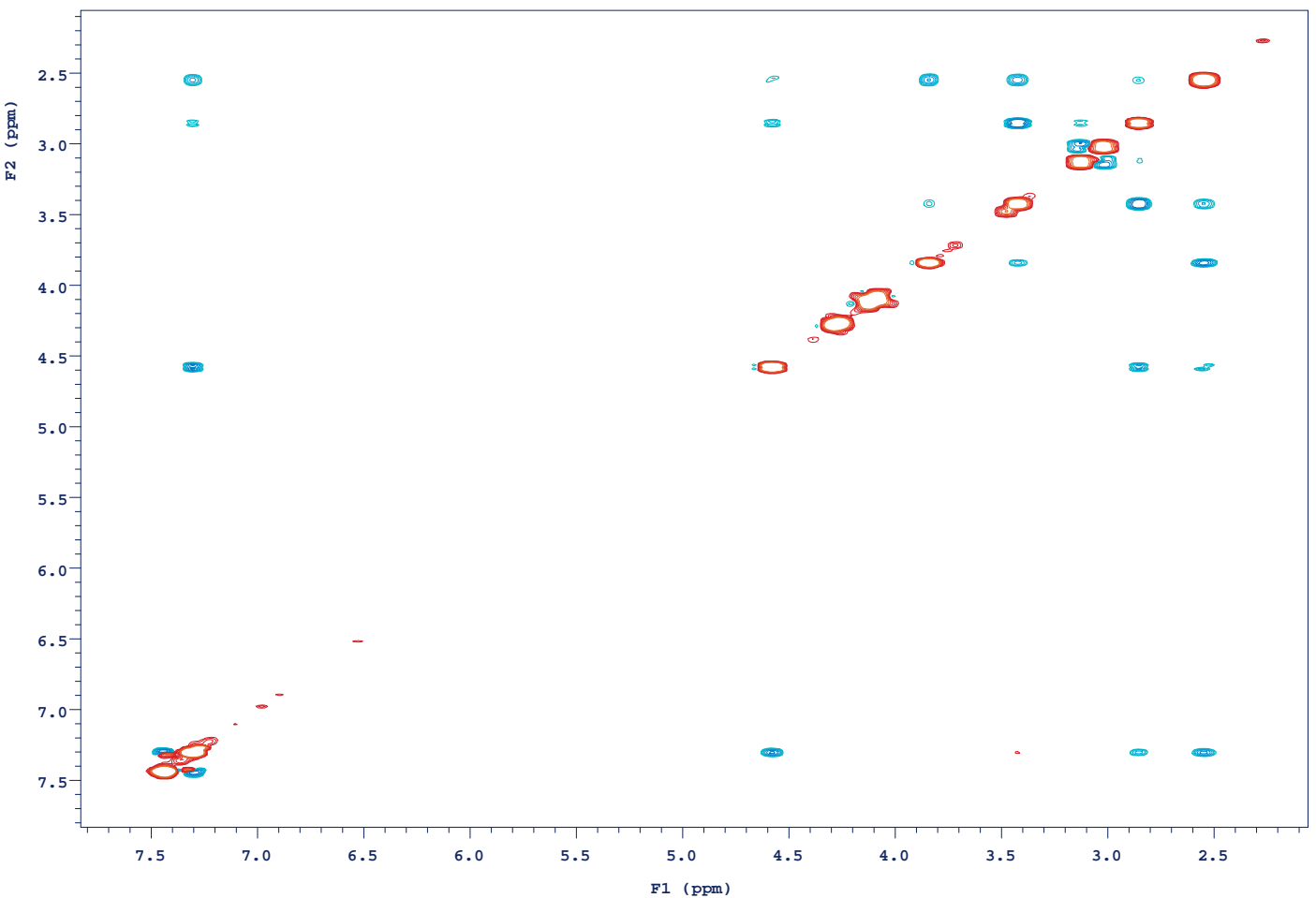



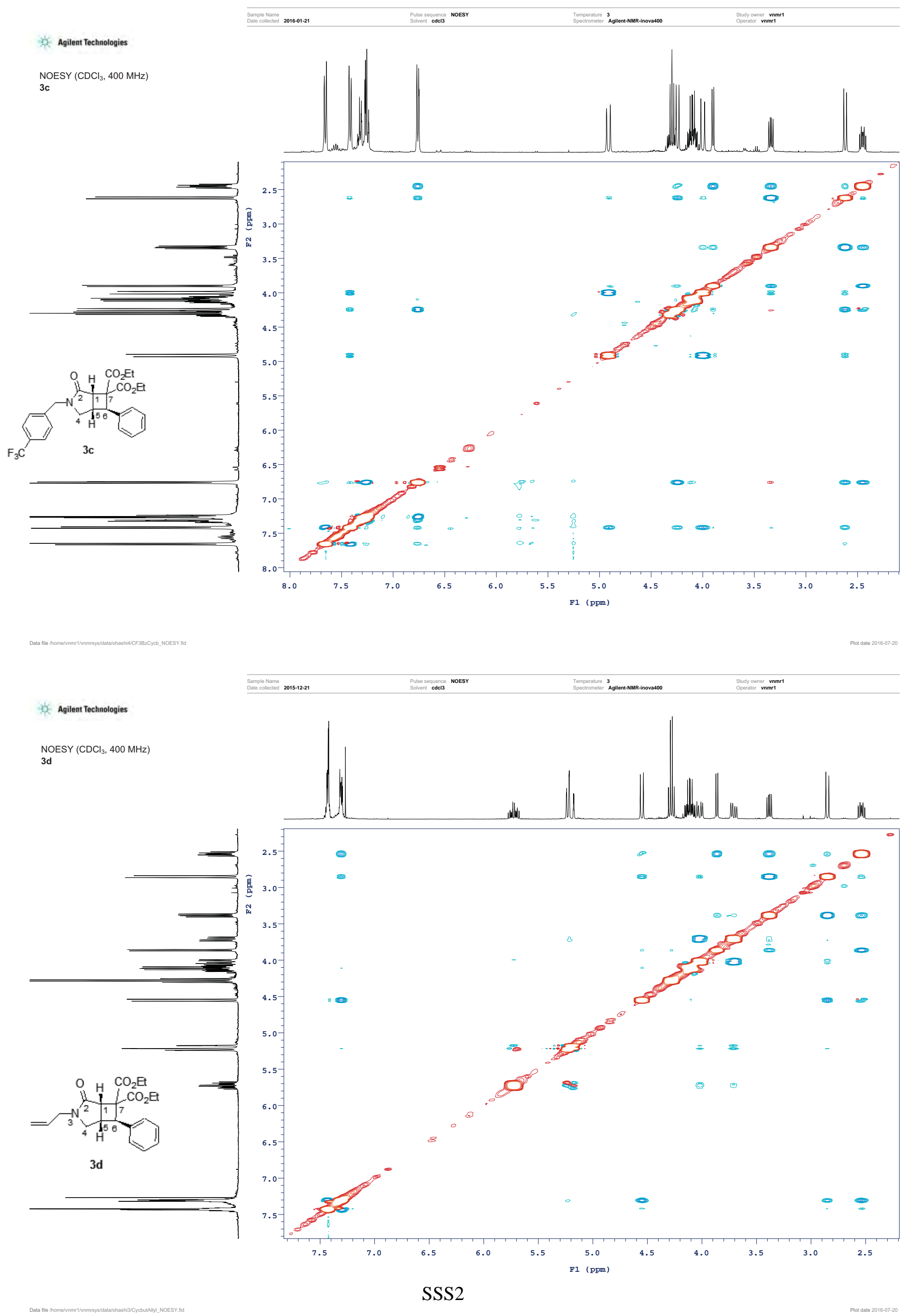

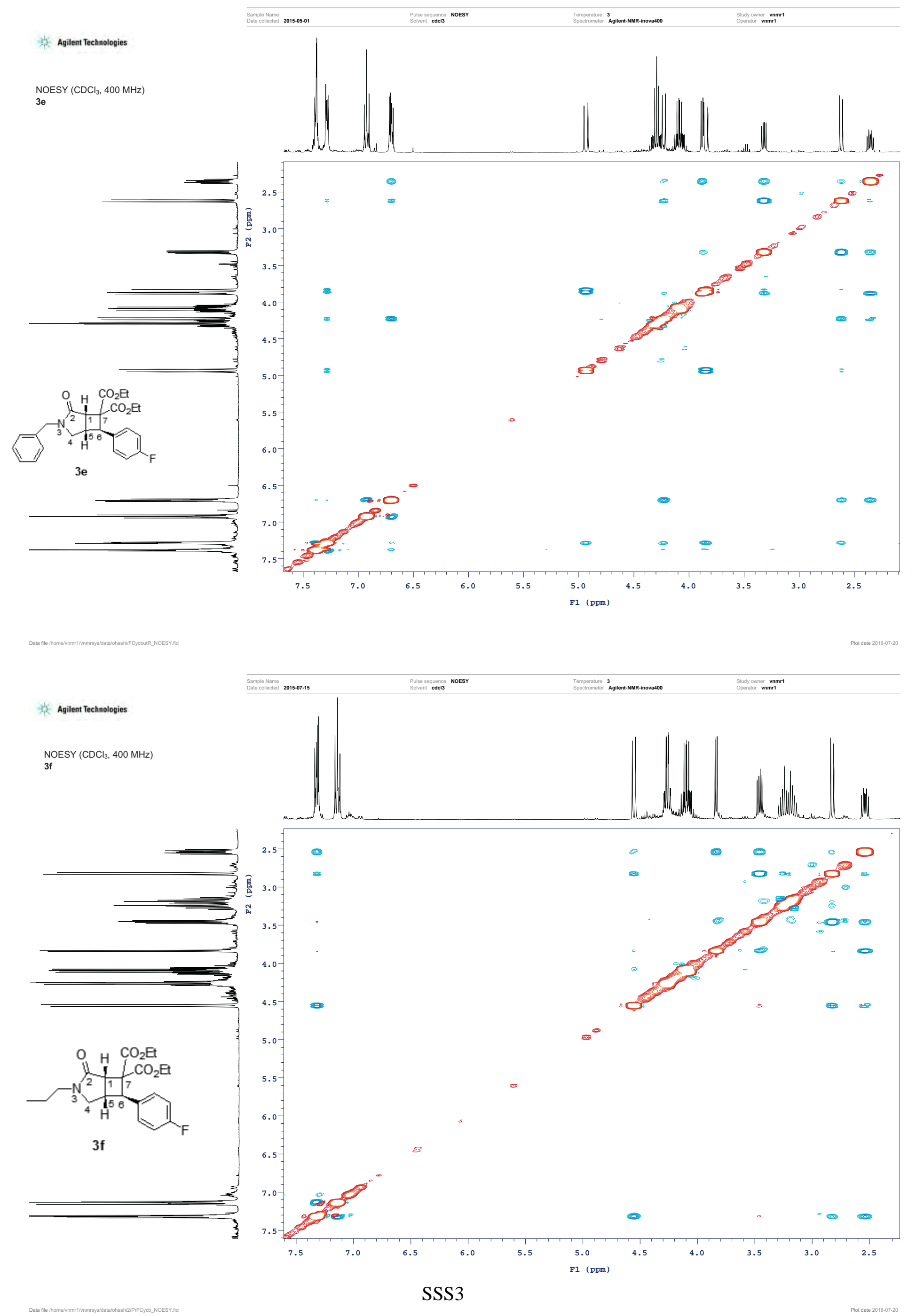

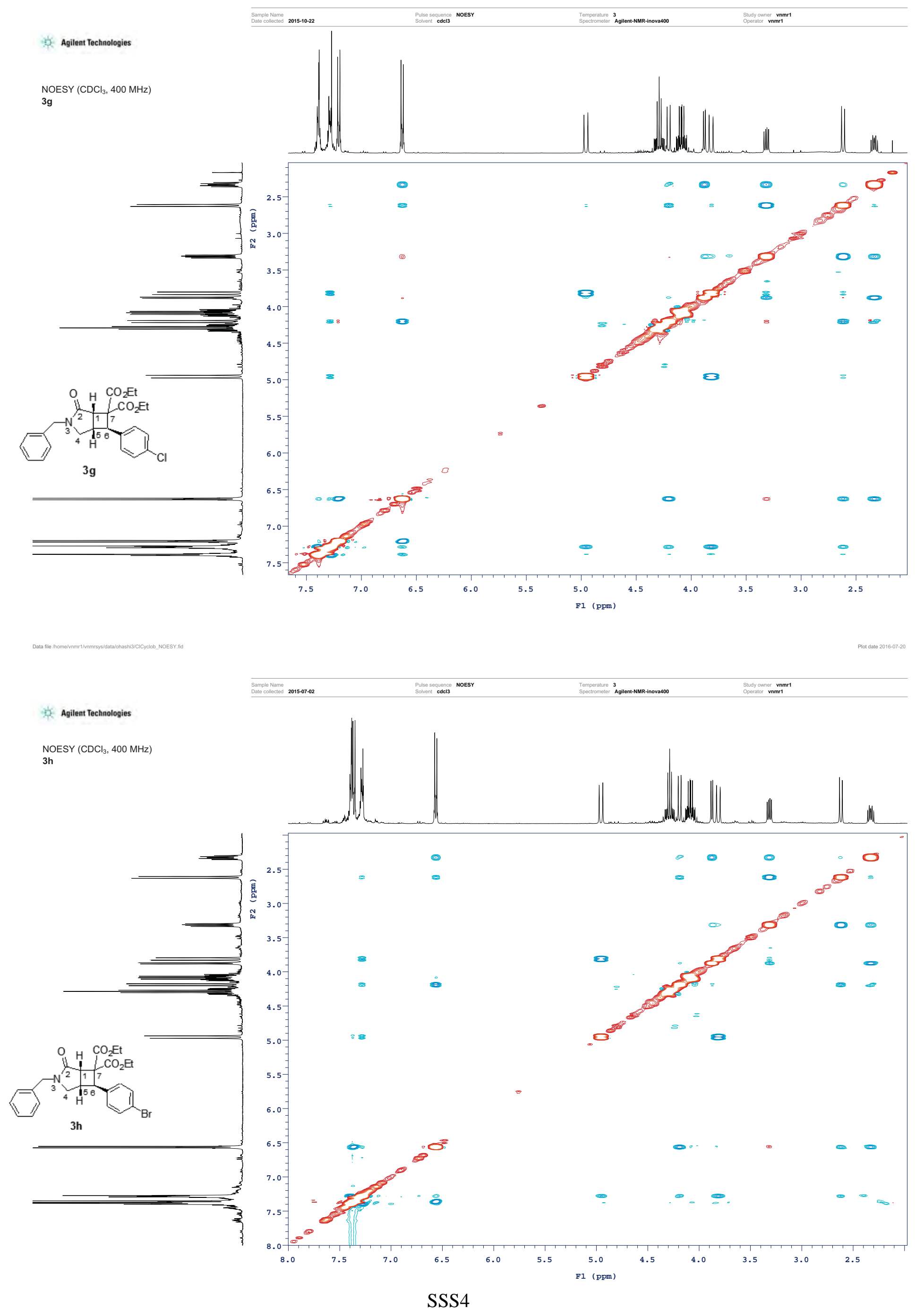

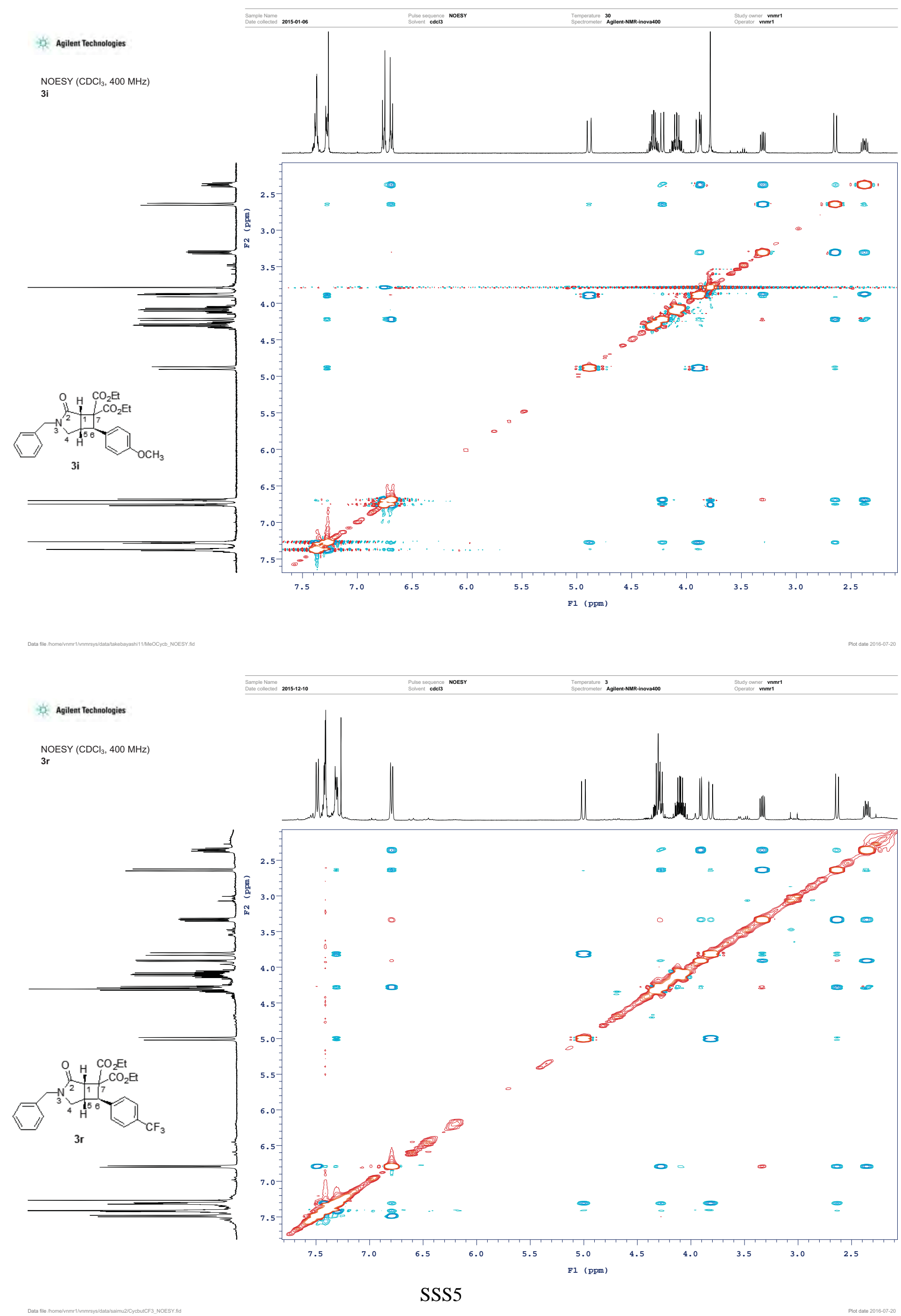

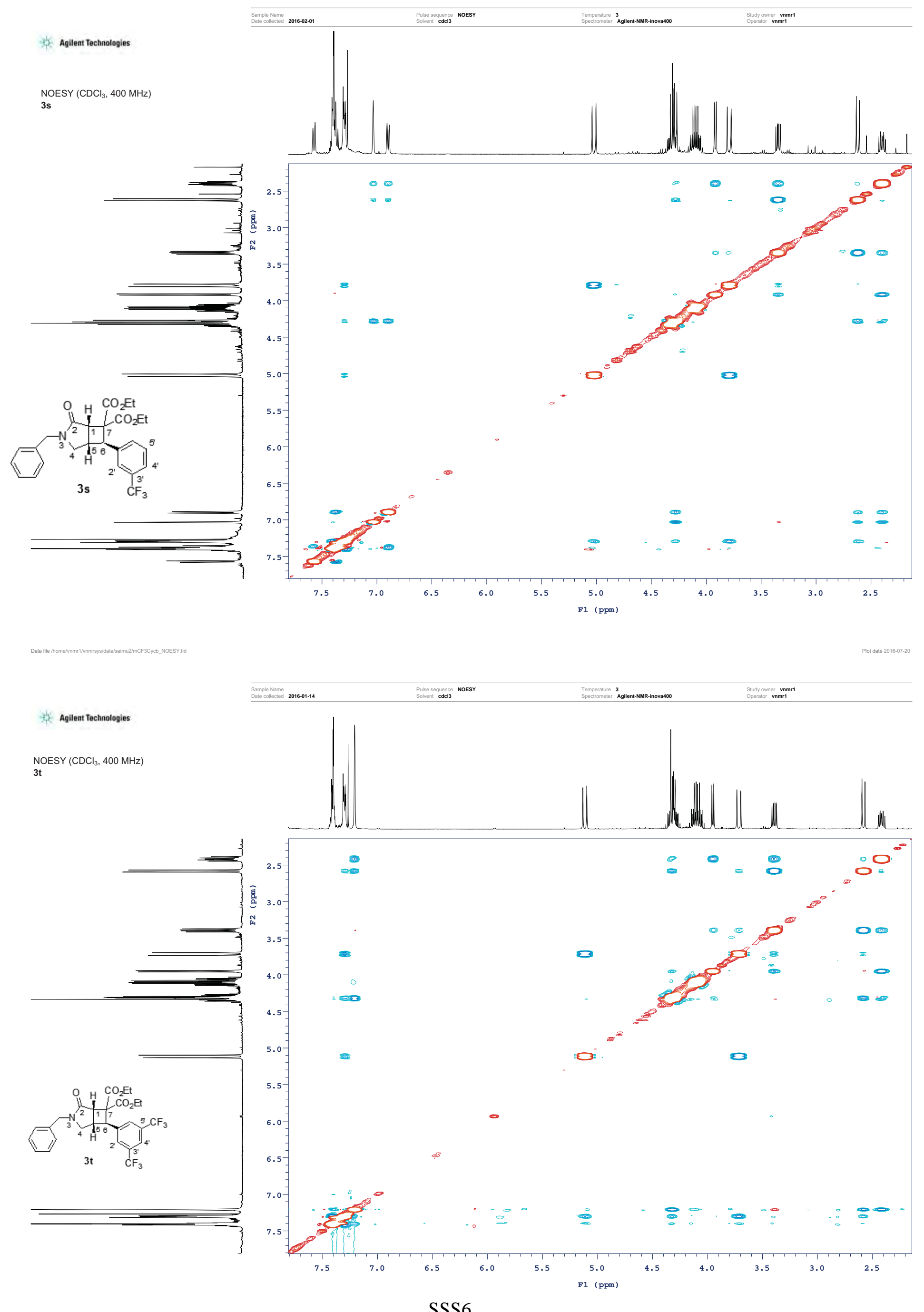

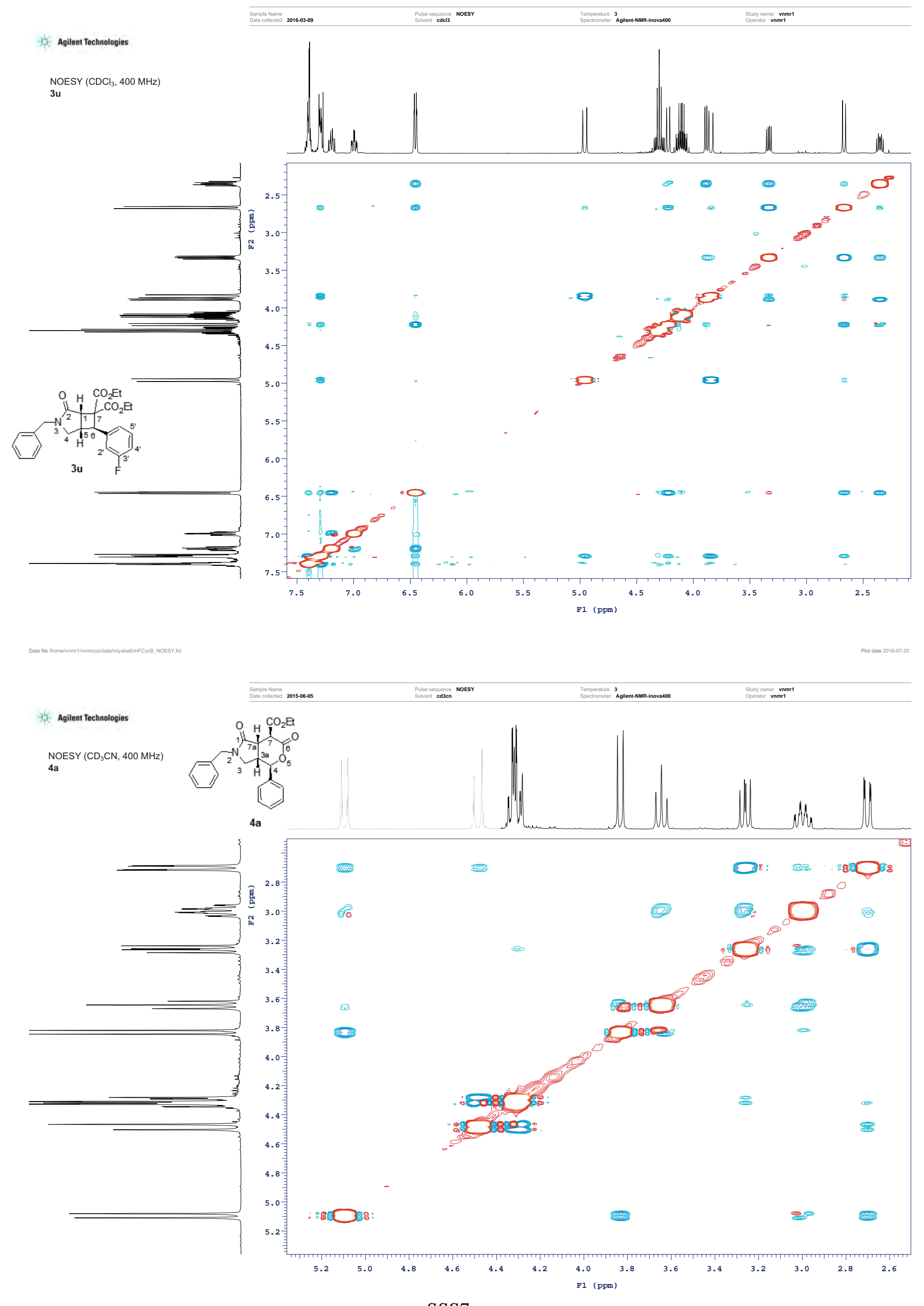


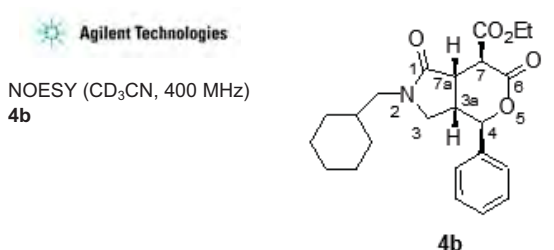

$4 b$
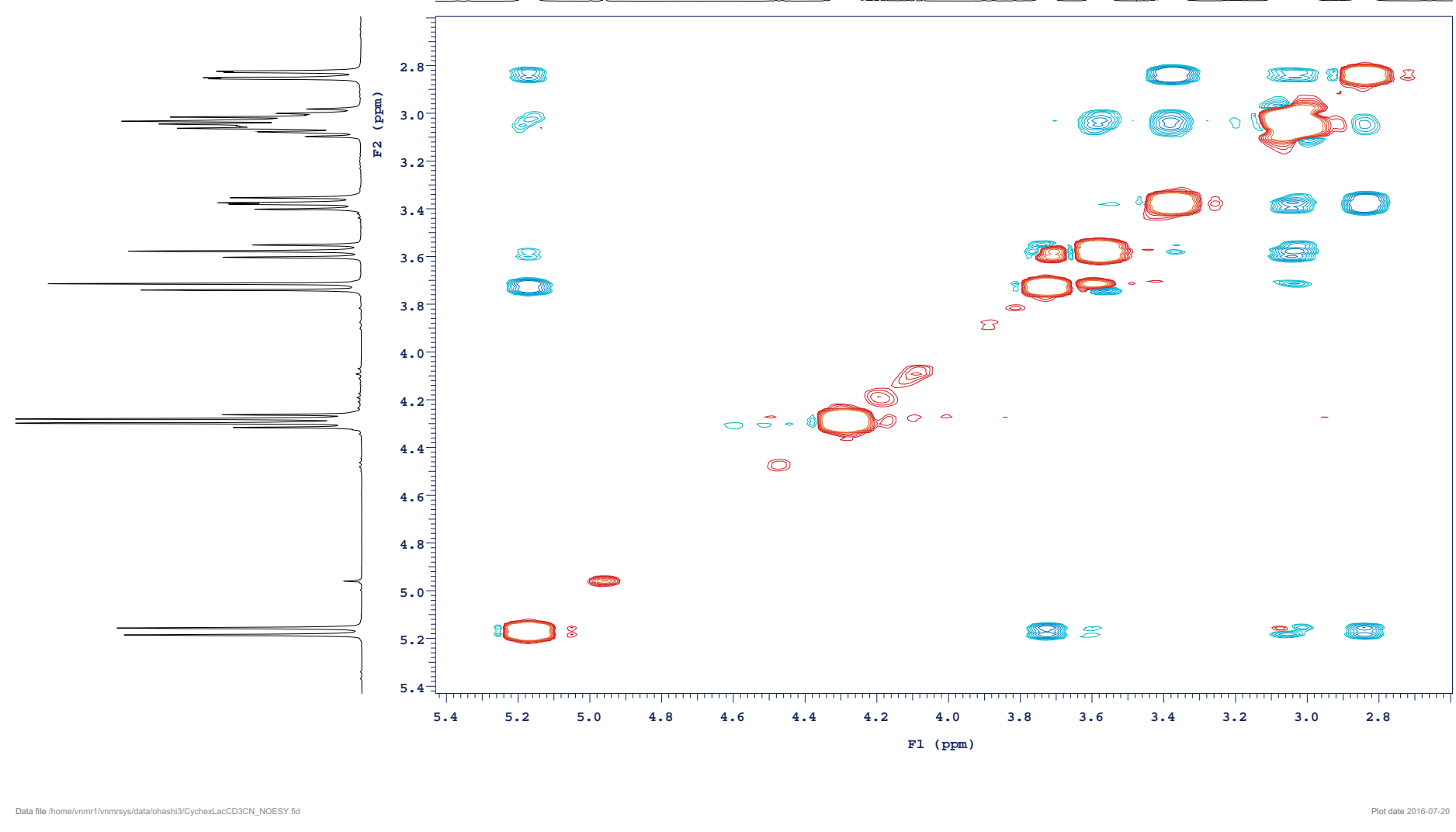

\begin{tabular}{|c|c|c|c|}
\hline $\begin{array}{l}\text { Sample Name } \\
\text { Date collected 2015-06-03 }\end{array}$ & $\begin{array}{l}\text { Pulse sequence NOESY } \\
\text { Solvent acetone }\end{array}$ & $\begin{array}{l}\text { Temperature }{ }^{3} \\
\text { Spectrometer Agilent-NMR-inova } 400\end{array}$ & $\begin{array}{l}\text { Study owner vnmr1 } \\
\text { Operator vnmr1 }\end{array}$ \\
\hline
\end{tabular}

\section{Agilent Technologies}

NOESY $\left(\left(\mathrm{CD}_{3}\right)_{2} \mathrm{CO}, 400 \mathrm{MHz}\right)$

$4 \mathrm{e}$

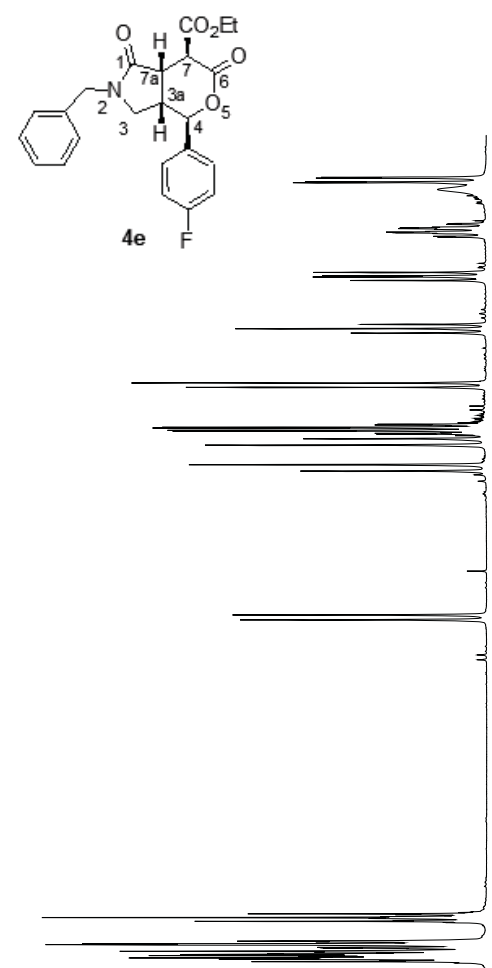

meter Agilent-NMR-inova400

Sudy owner vnmr
Oevator vnmr1

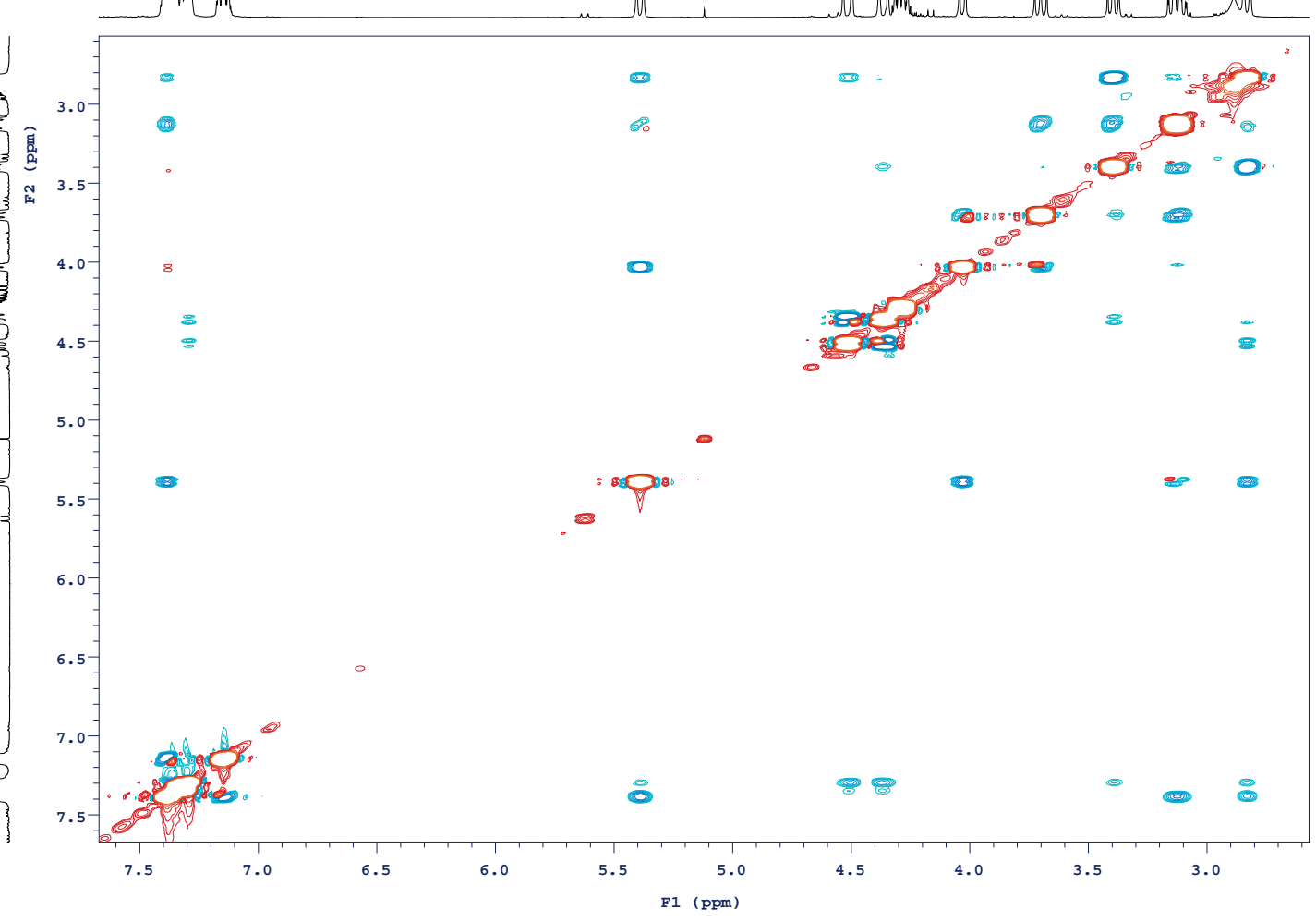



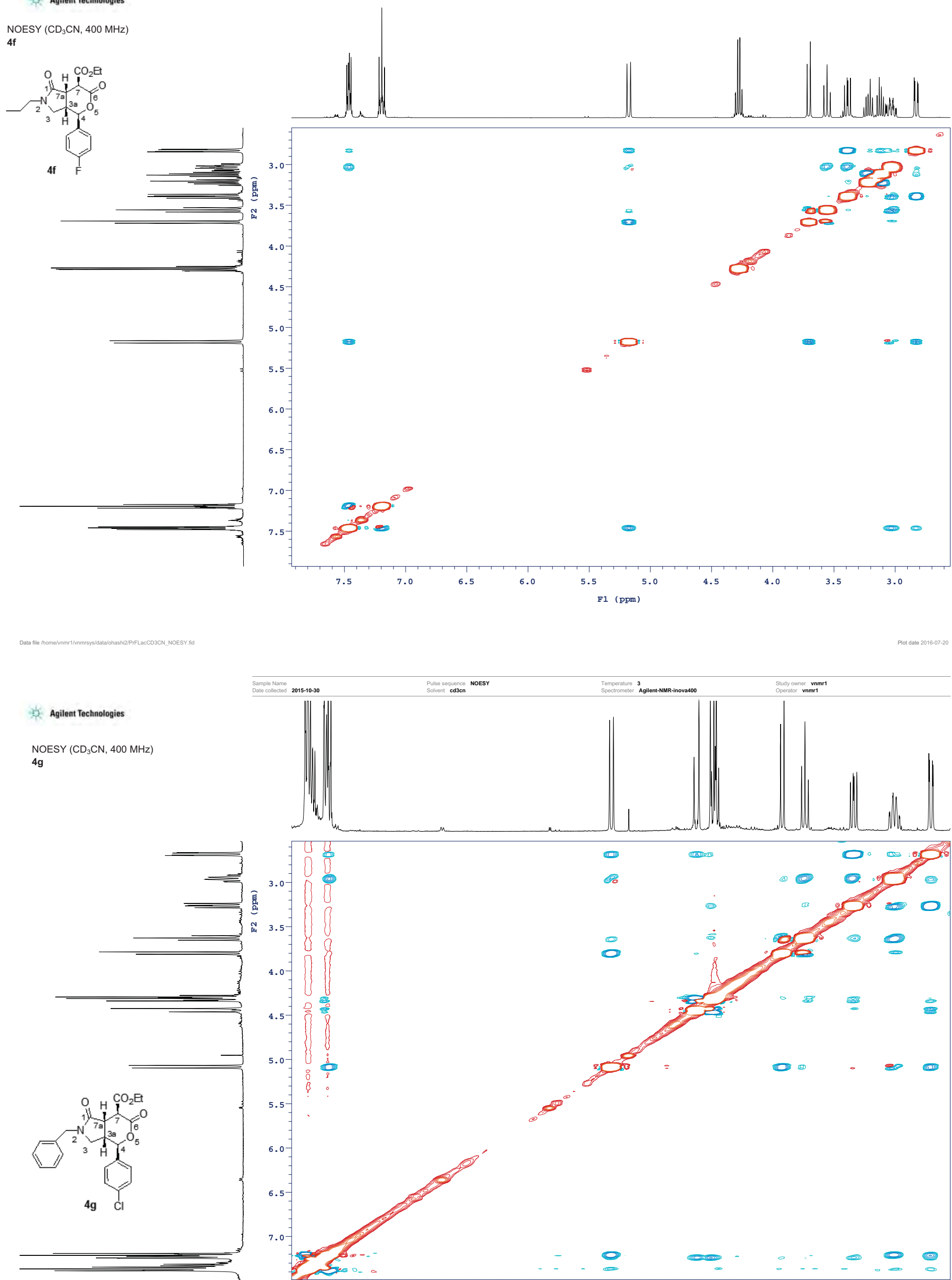
NOESY $\left(\mathrm{CD}_{3} \mathrm{CN}, 400 \mathrm{MHz}\right)$

4h
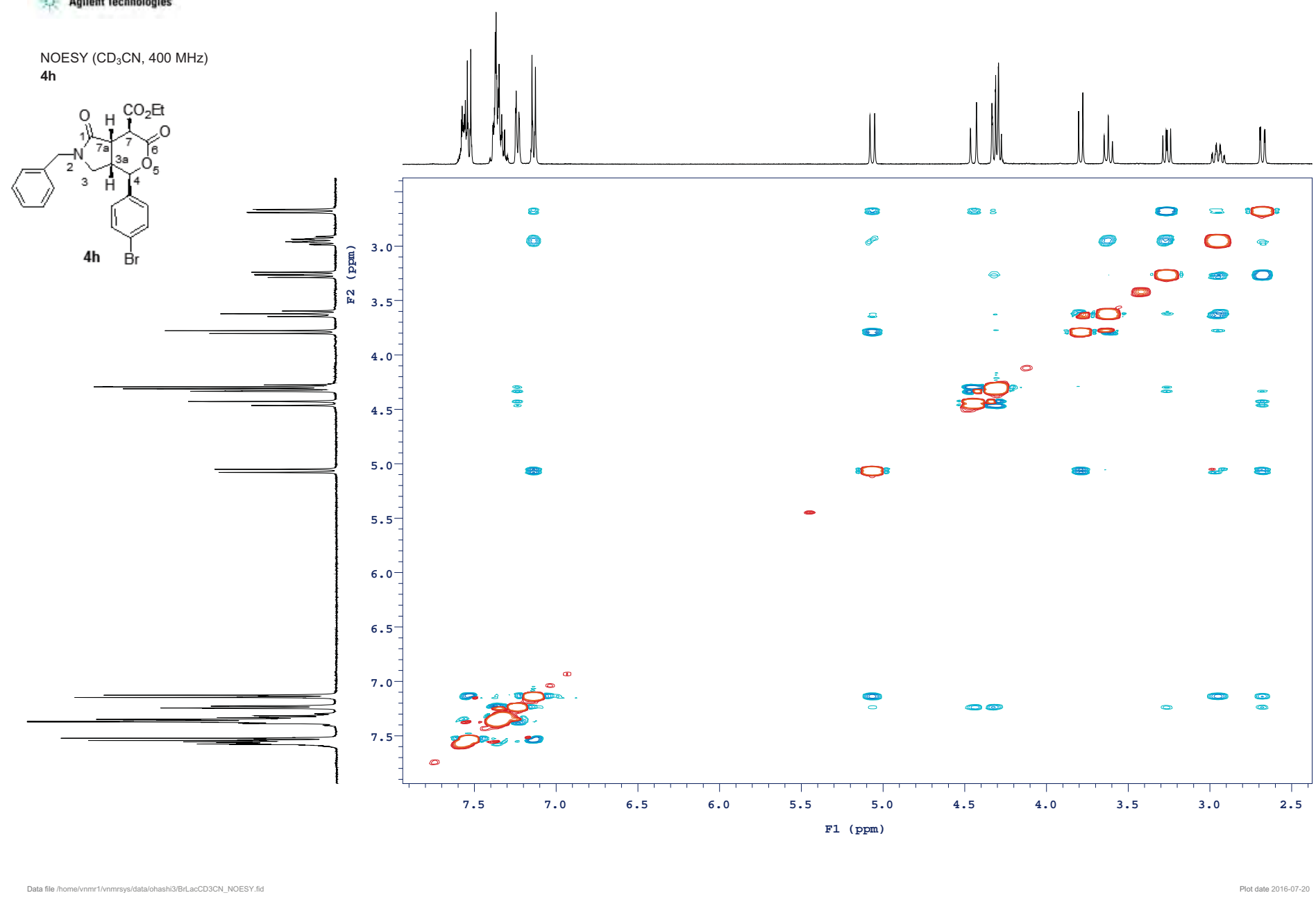

\section{Agilent Technologies}

NOESY $\left(\mathrm{CD}_{3} \mathrm{CN}, 400 \mathrm{MHz}\right)$

$4 i$

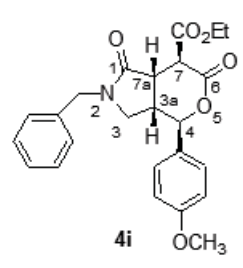

\begin{tabular}{ll}
\hline Sample Name $2015-09-19$ & $\begin{array}{l}\text { Puse sequence NOEST } \\
\text { Solvent cascr }\end{array}$ \\
\hline Date collectod 20
\end{tabular}

Temperature
Spectrometer
Agilent-NMR-inovatoo

Study owner vnmr1
Operator vnmr1
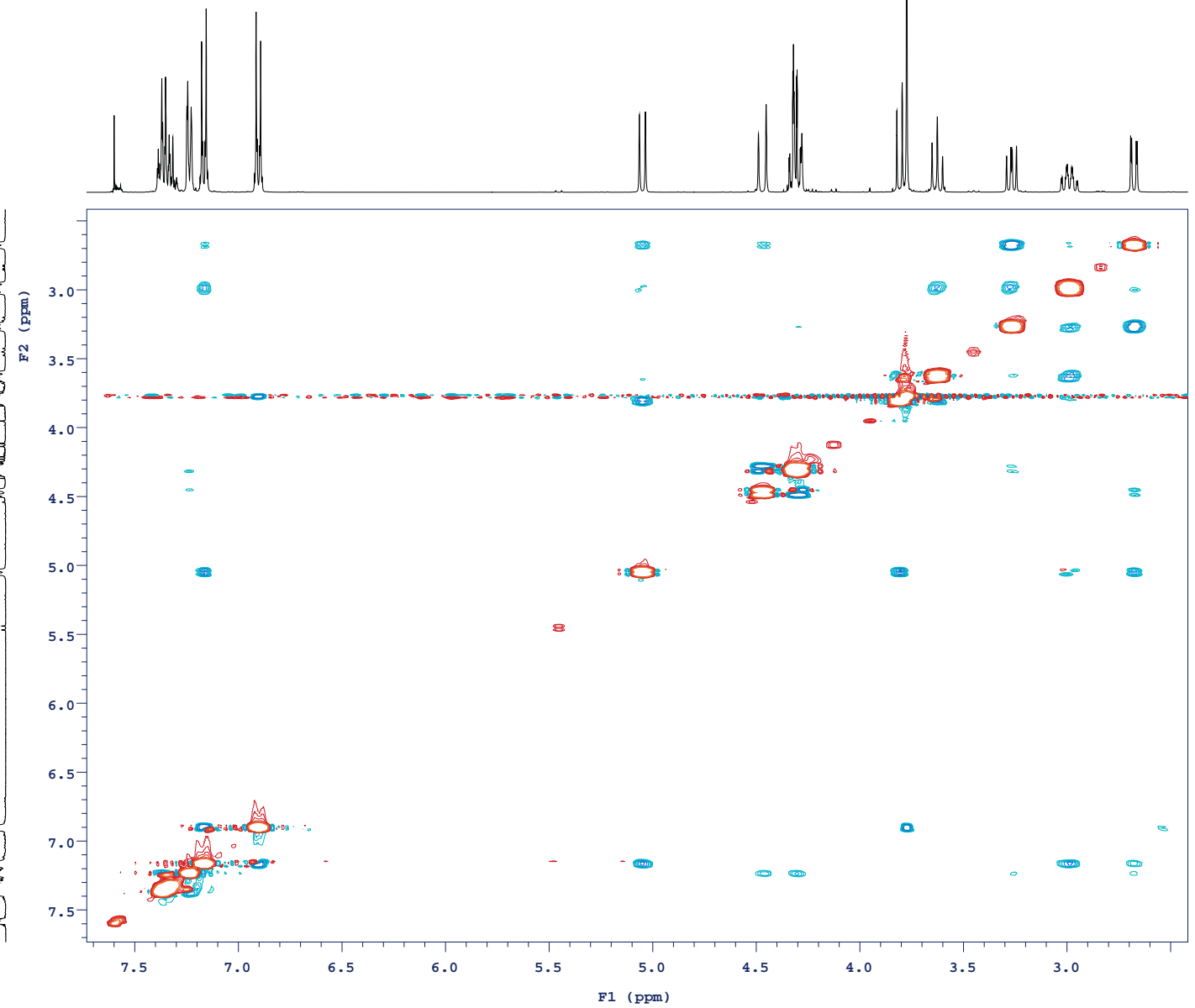


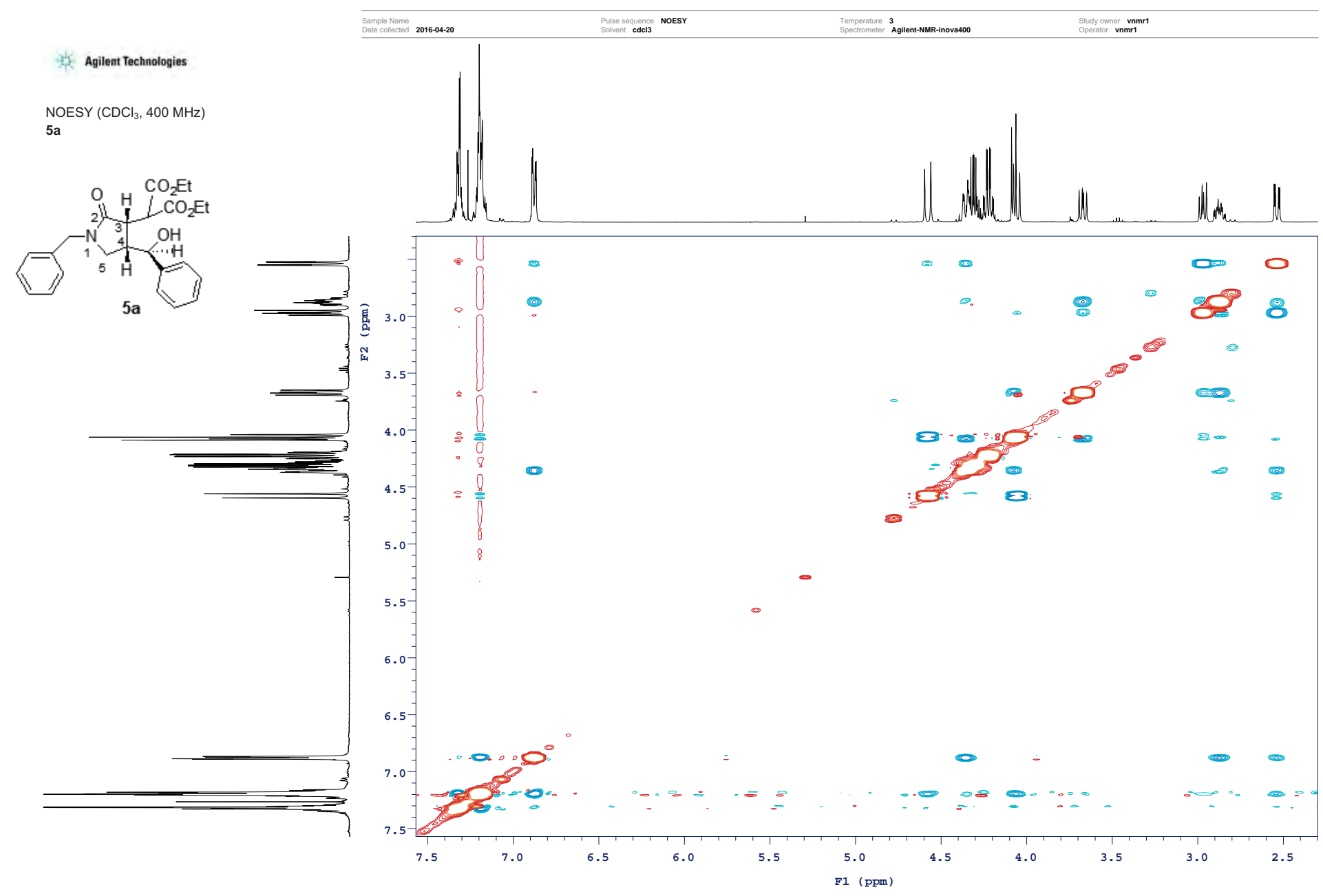

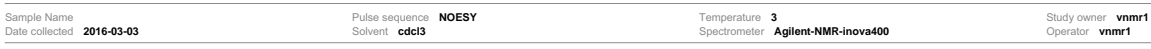
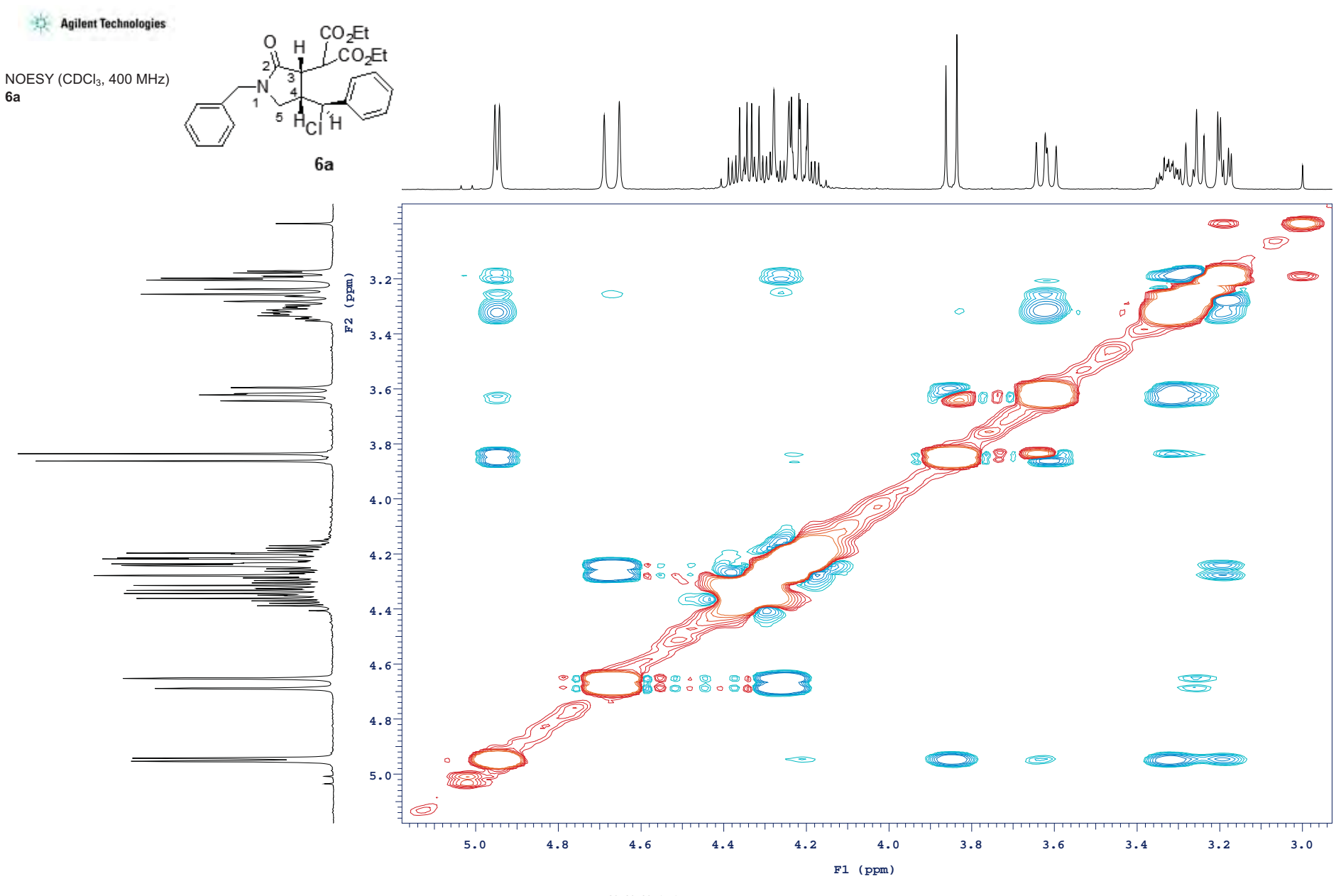

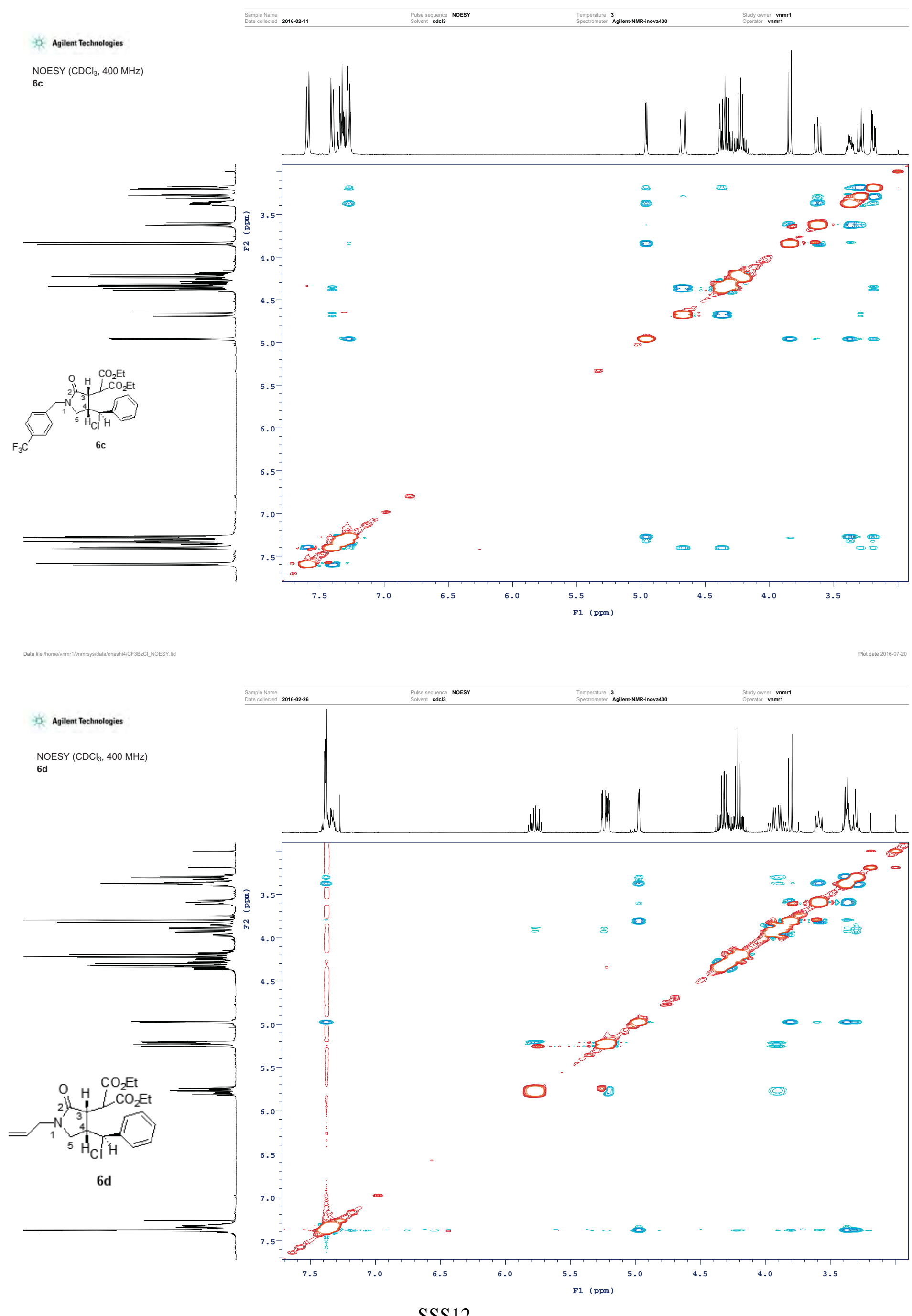


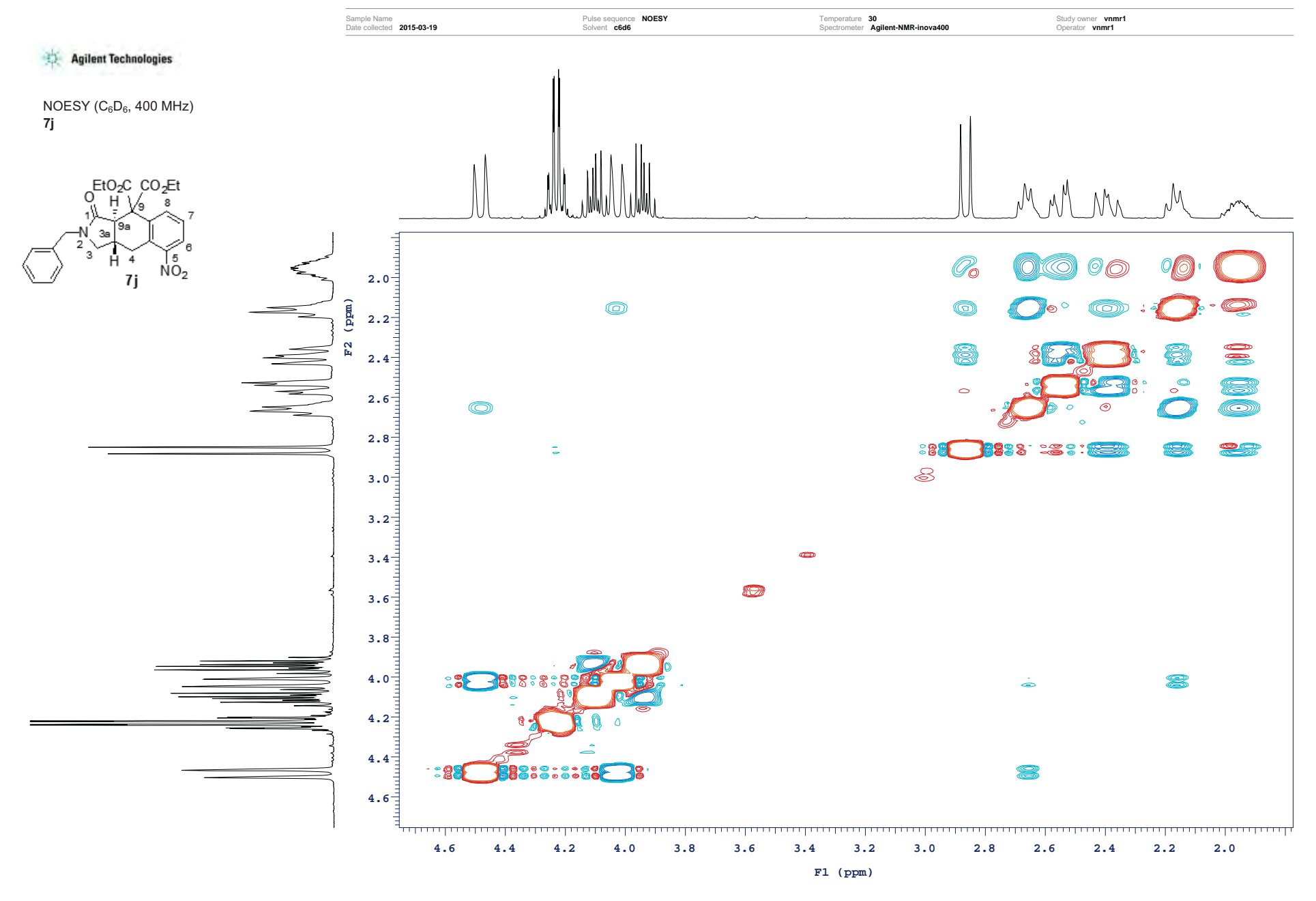

\begin{tabular}{|c|c|c|c|}
\hline $\begin{array}{l}\text { Sample Name } \\
\text { Date collected 2015-09-17 }\end{array}$ & $\begin{array}{l}\text { Pulse sequence NOESY } \\
\text { Solvent codd6 }\end{array}$ & $\begin{array}{l}\text { Temperature }{ }^{3} \\
\text { Spectrometer Agilent-NMR-inova400 }\end{array}$ & $\begin{array}{l}\text { Study owner vnmr1 } \\
\text { Operator vnmr1 }\end{array}$ \\
\hline
\end{tabular}

3. Agilent Technologies

$\operatorname{NOESY}\left(\mathrm{C}_{6} \mathrm{D}_{6}, 400 \mathrm{MHz}\right)$

$7 k$
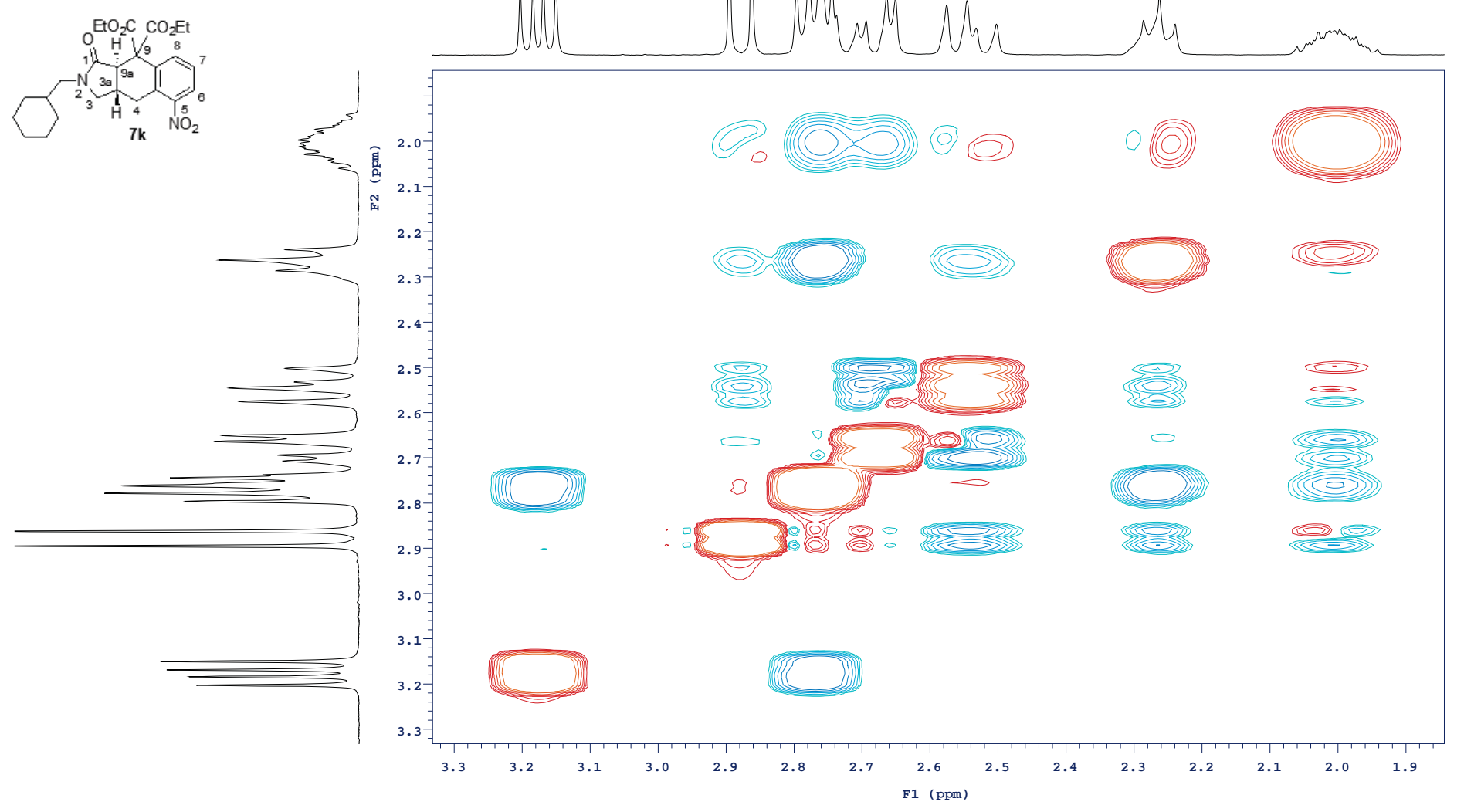

SSS13 

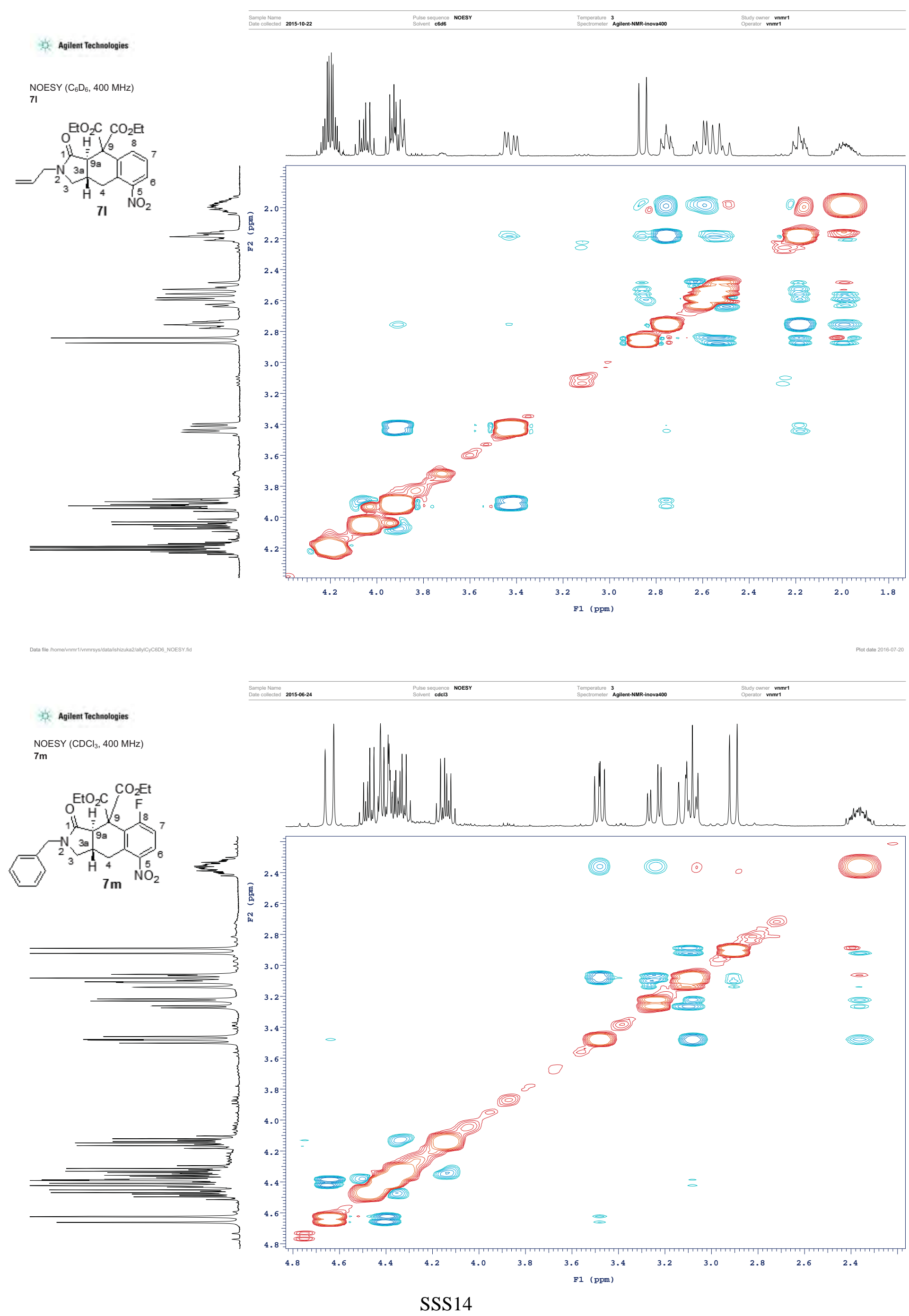
NOESY $\left(\left(\mathrm{CD}_{3}\right)_{2} \mathrm{CO}, 400 \mathrm{MHz}\right)$ $7 n$
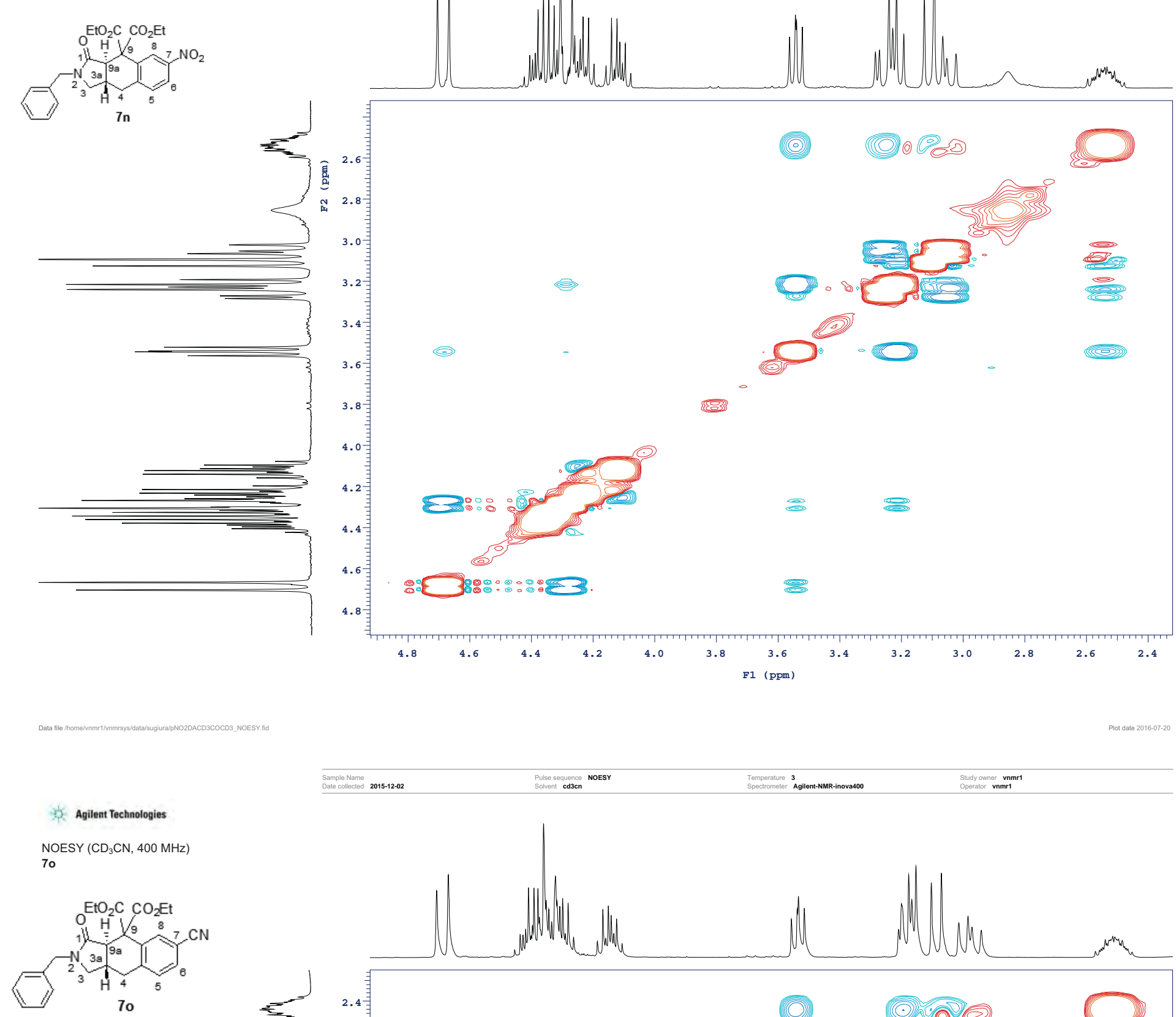

NOESY $\left(\mathrm{CD}_{3} \mathrm{CN}, 400 \mathrm{MHz}\right)$

$\mathrm{EtO}_{2} \mathrm{C} \mathrm{CO} \mathrm{Ct}_{2}$

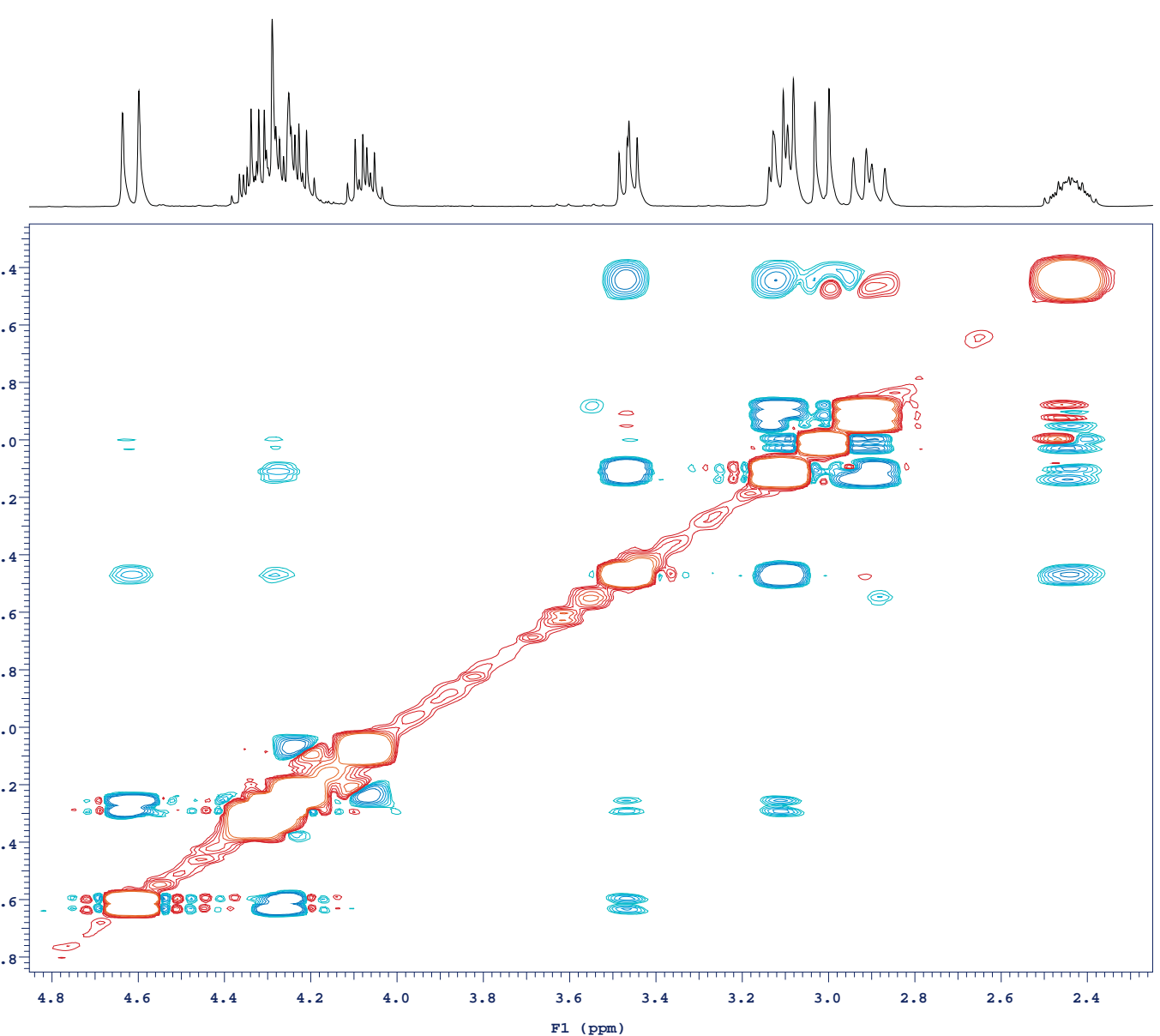




\section{कf. Agilent Technologies}

NOESY $\left(\mathrm{CD}_{3} \mathrm{CN}, 400 \mathrm{MHz}\right)$ $7 \mathrm{p}$
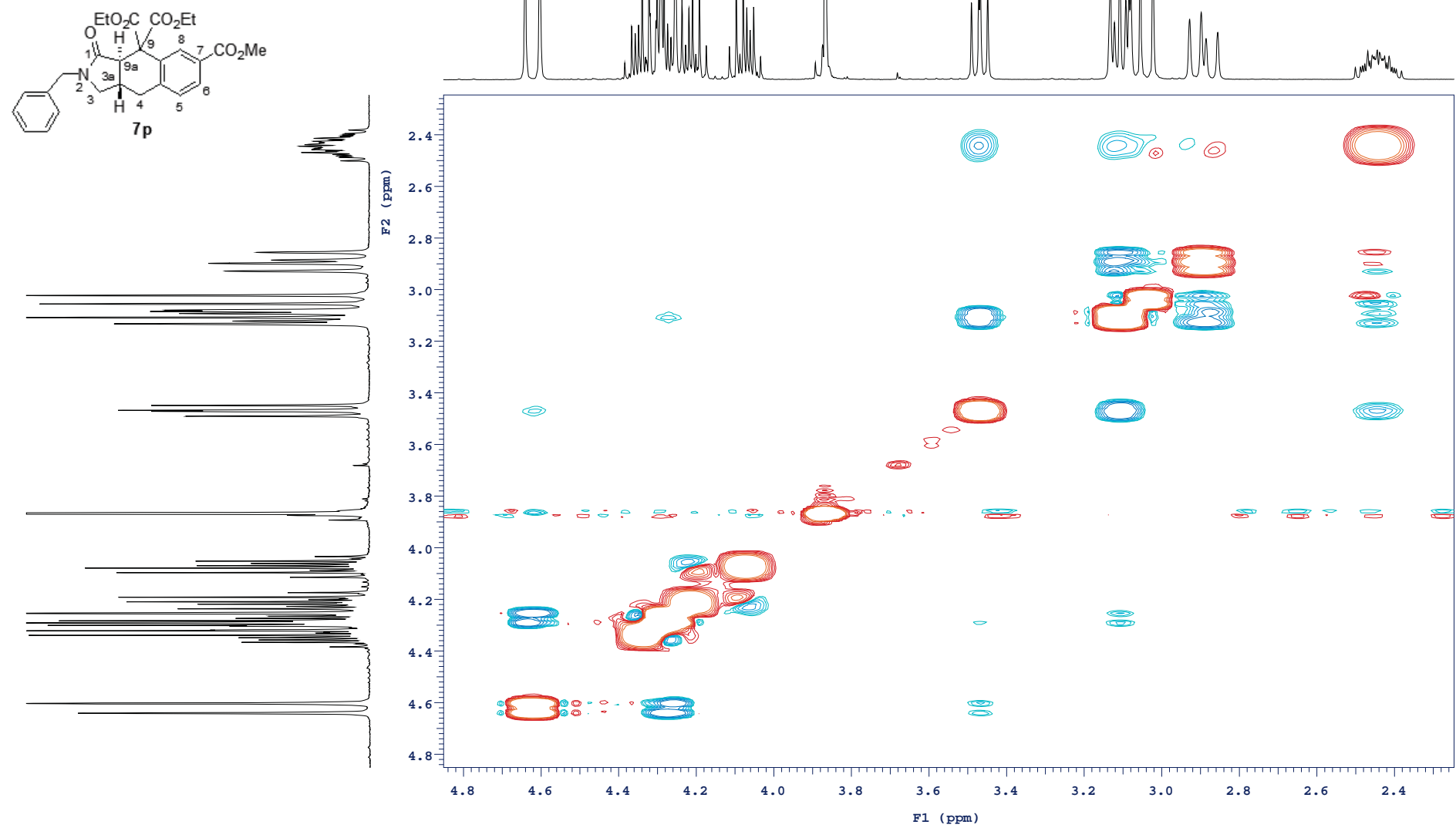

\section{Agilent Technologies}

NOESY $\left(\mathrm{CD}_{3} \mathrm{CN}, 400 \mathrm{MHz}\right)$

7q

\section{Sample Name
Date collected 2016-02-26}

Pulse sequence NOESY
Solvent cal3n

Temperature
Spectrometer ${ }^{3}$ Agilent-NMR-inova400

Study owner vnmrn
Opperator vnnr1
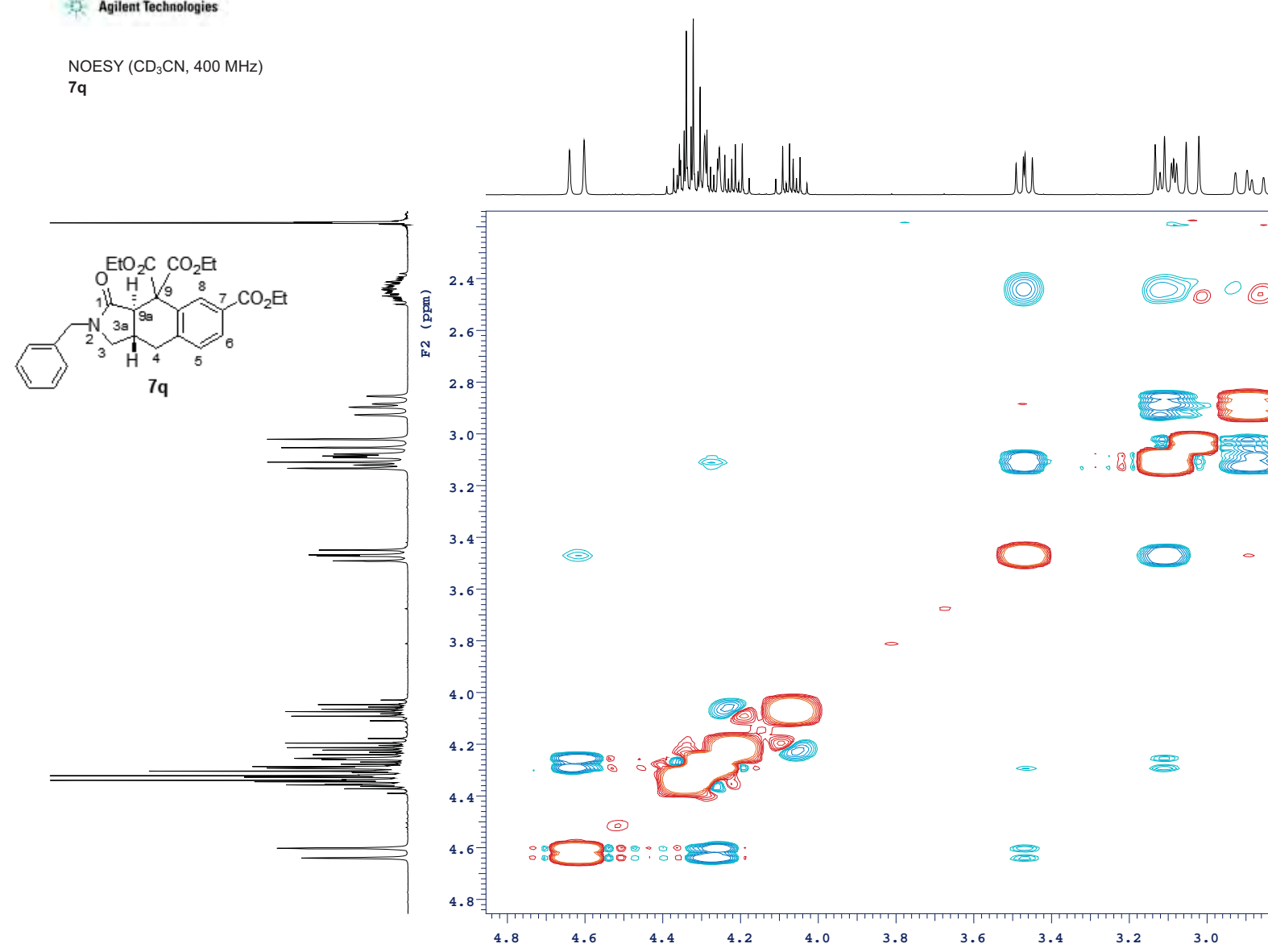

\section{SSS16}


NOESY $\left(\mathrm{CD}_{3} \mathrm{CN}, 400 \mathrm{MHz}\right)$ $7 \mathrm{r}$
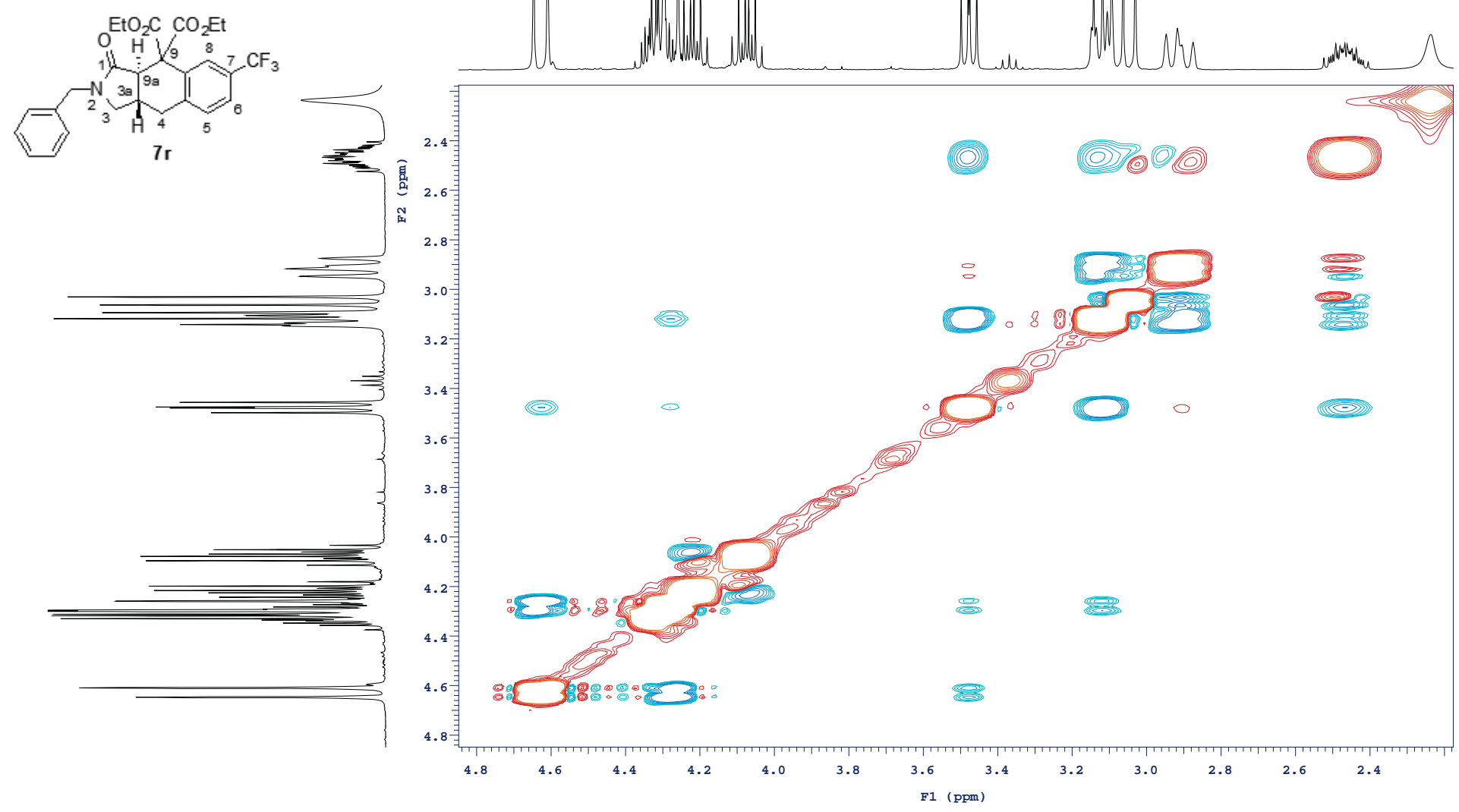

\section{Agilent Technologies}

NOESY $\left(\mathrm{CD}_{3} \mathrm{CN}, 400 \mathrm{MHz}\right)$

$7 \mathrm{~s}$
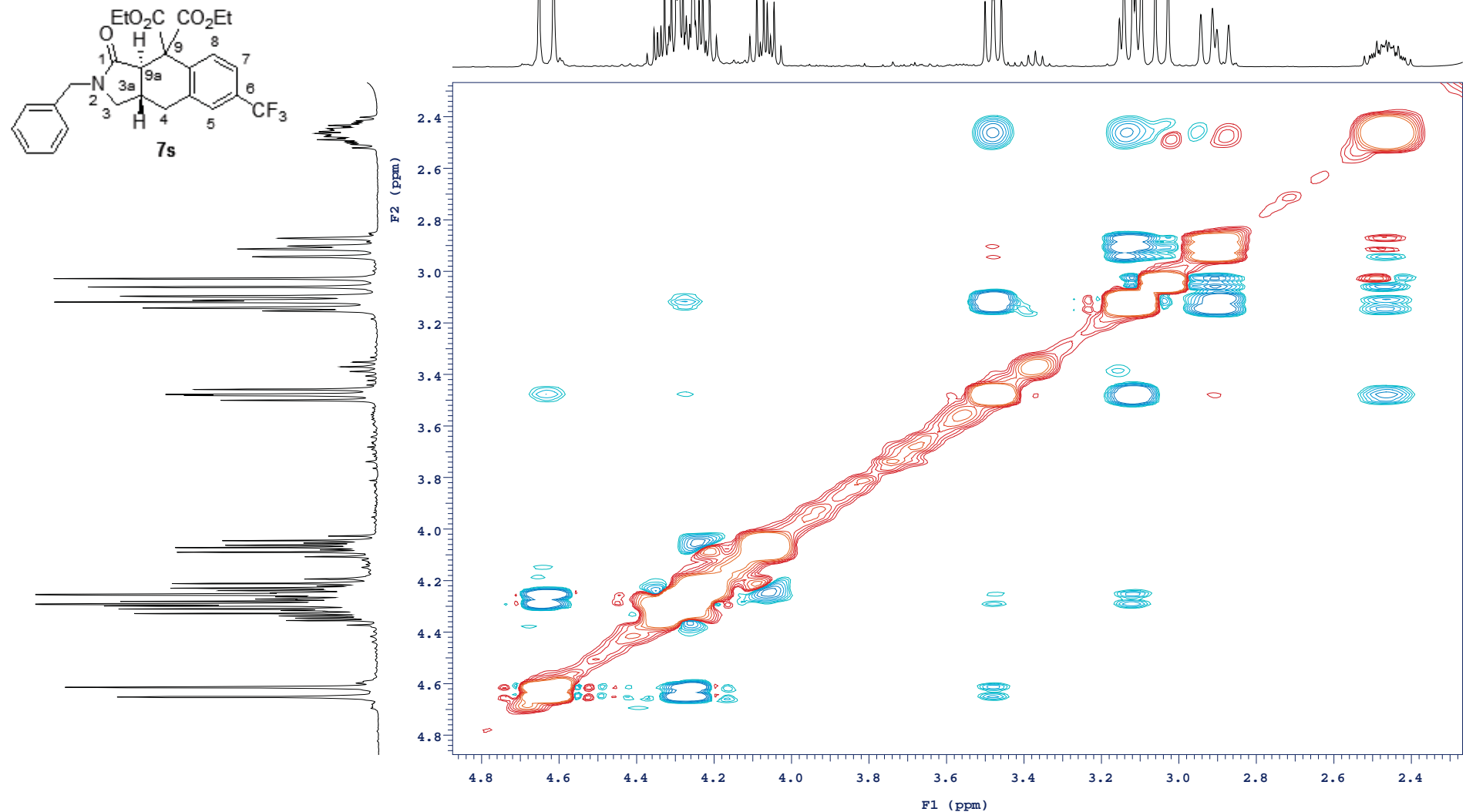
$\mathrm{NOESY}\left(\mathrm{CDCl}_{3}, 400 \mathrm{MHz}\right)$

6-F-7u/8-F-7u

$\mathrm{EtO}_{2} \mathrm{C} \mathrm{CO}_{2} \mathrm{Et}$

1)

$\mathrm{CHO}_{2} \mathrm{C} \mathrm{CO}_{2} \mathrm{Et}$

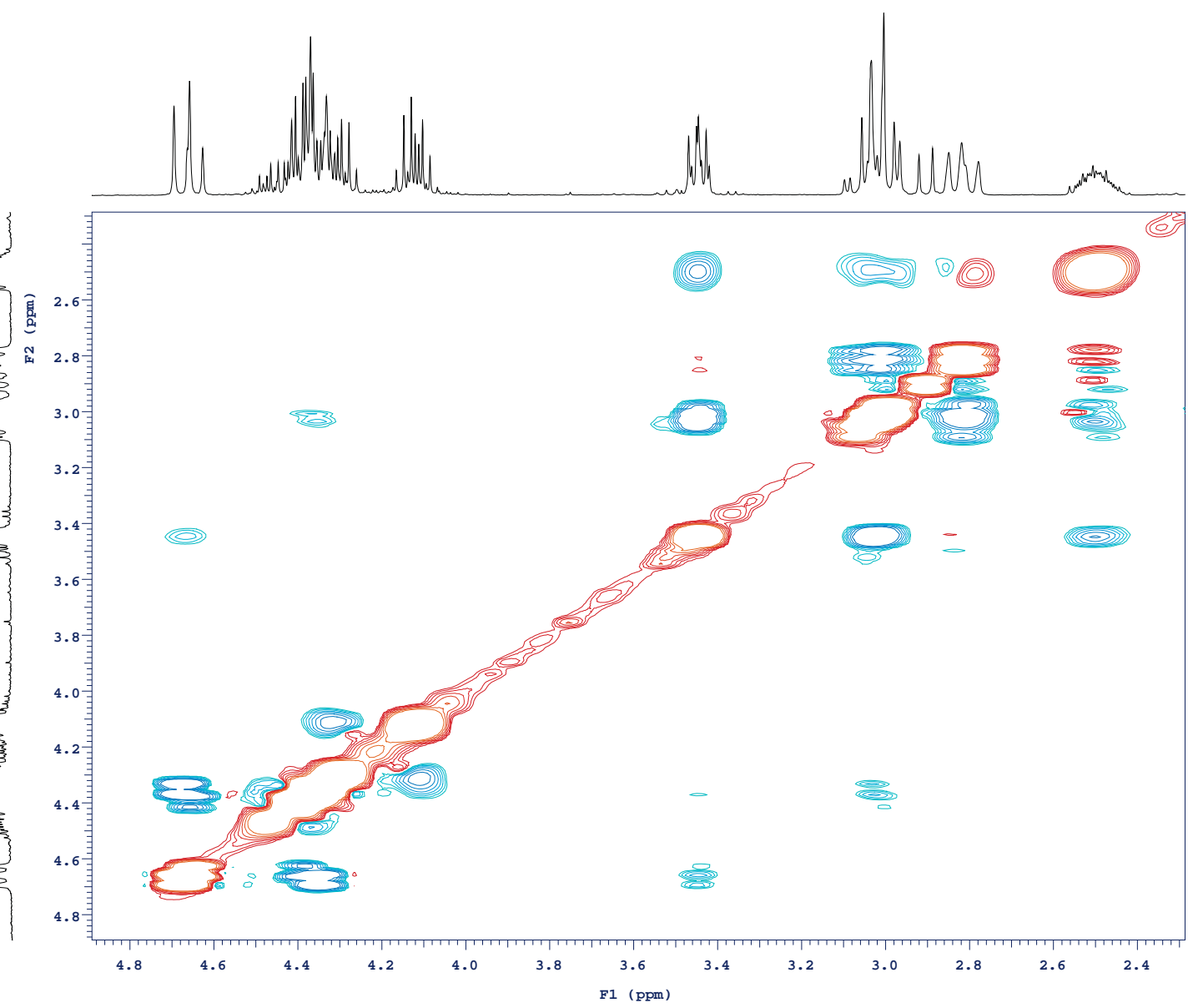

6-F-7u

(2.5

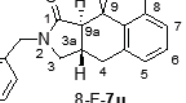

$8-\mathrm{F}-7 \mathrm{u}$
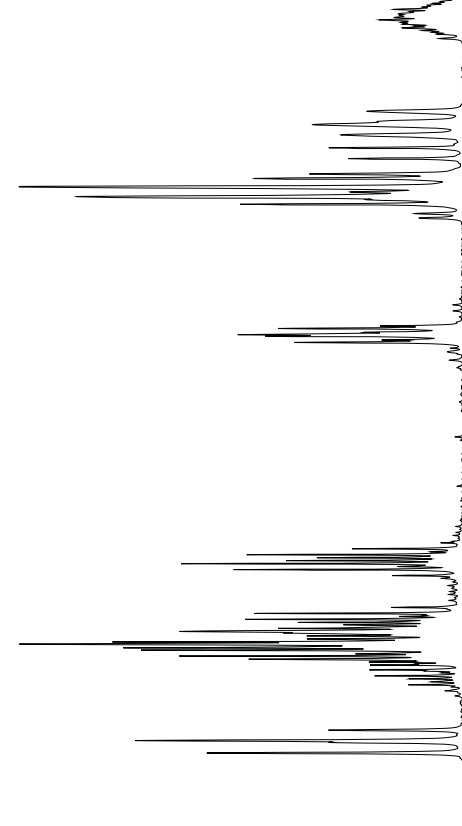

\section{के Agilent Technologies}

NOESY $\left(\mathrm{C}_{6} \mathrm{D}_{6}, 400 \mathrm{MHz}\right)$
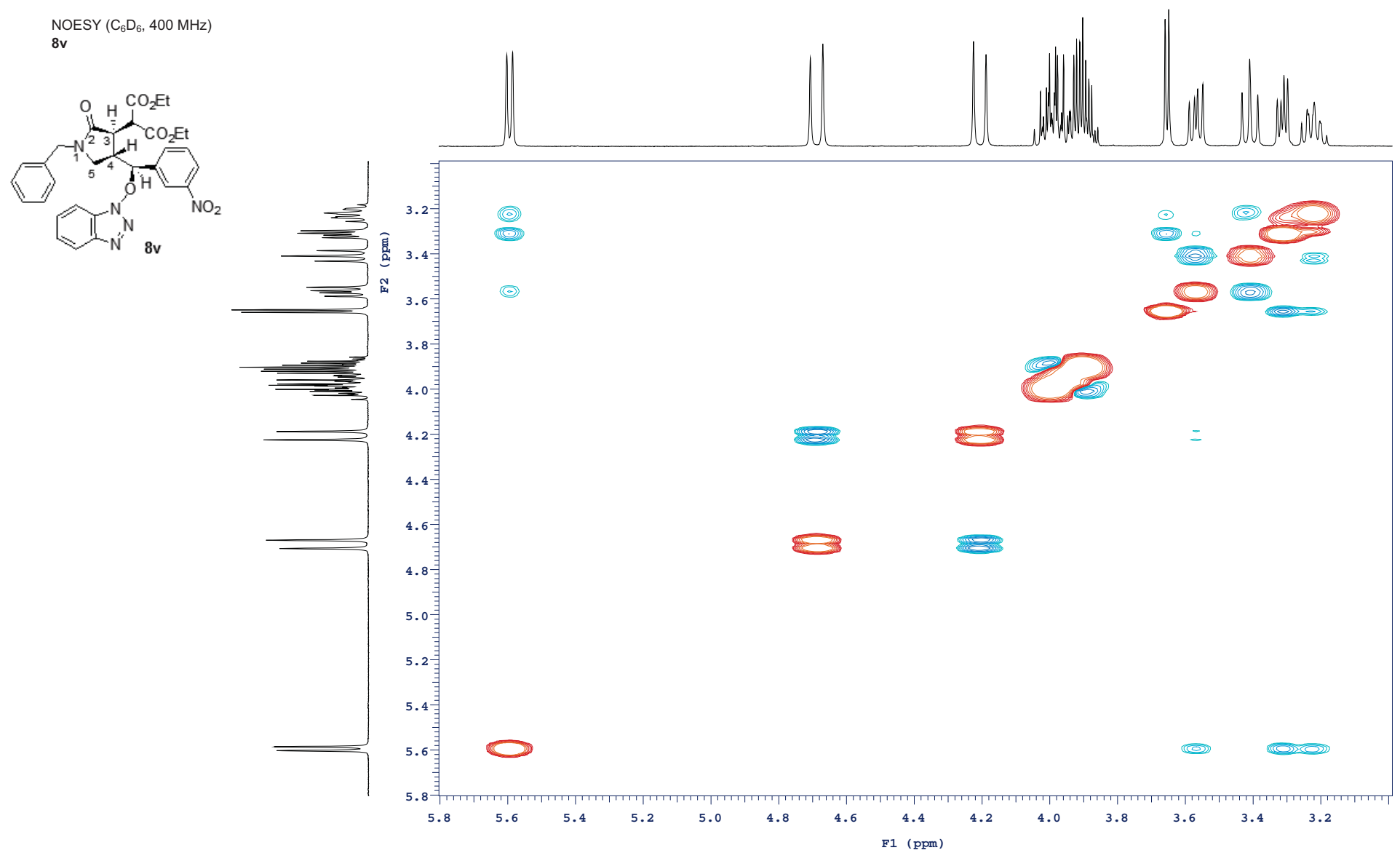

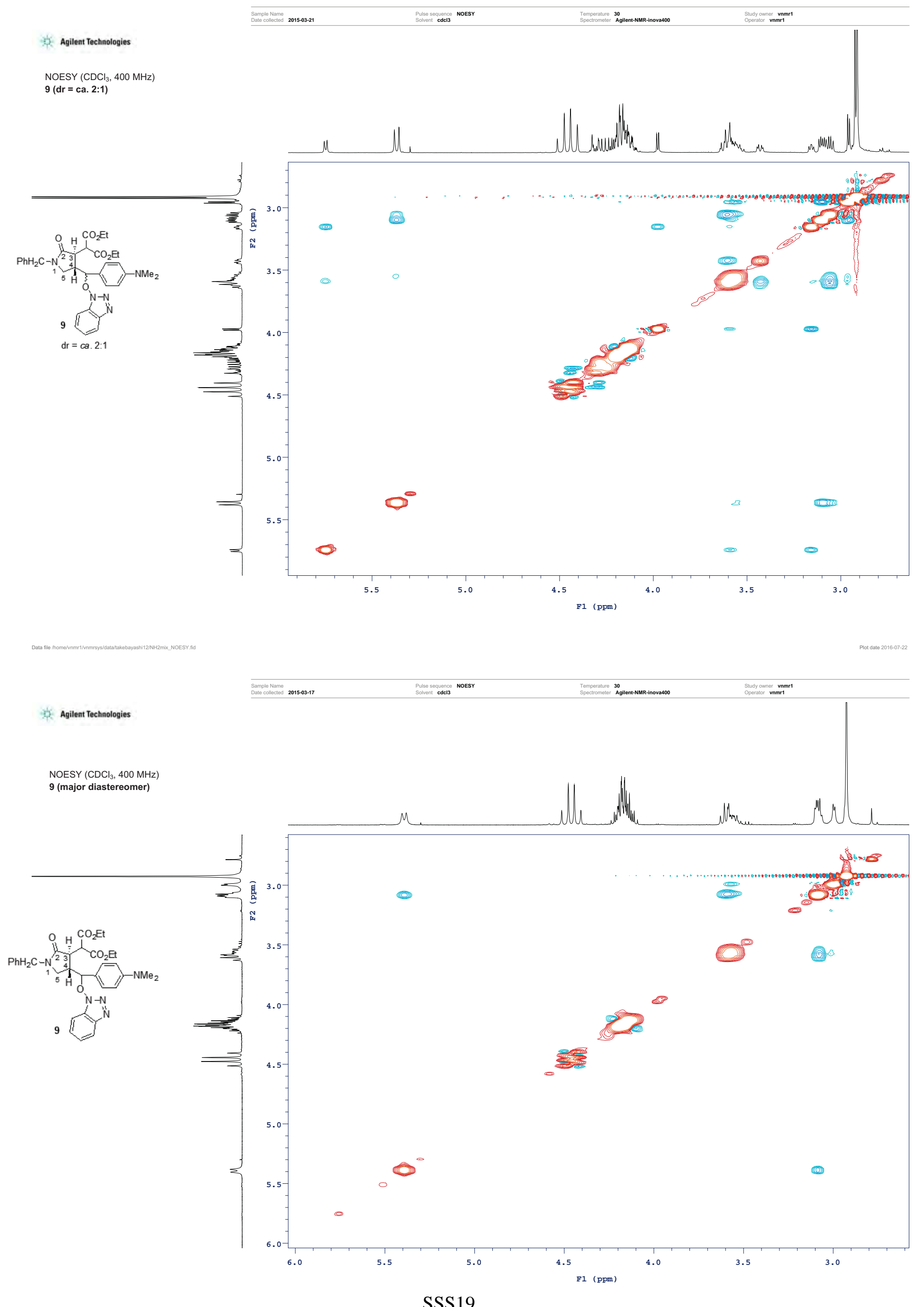
$\mathrm{NOESY}\left(\mathrm{CDCl}_{3}, 400 \mathrm{MHz}\right)$
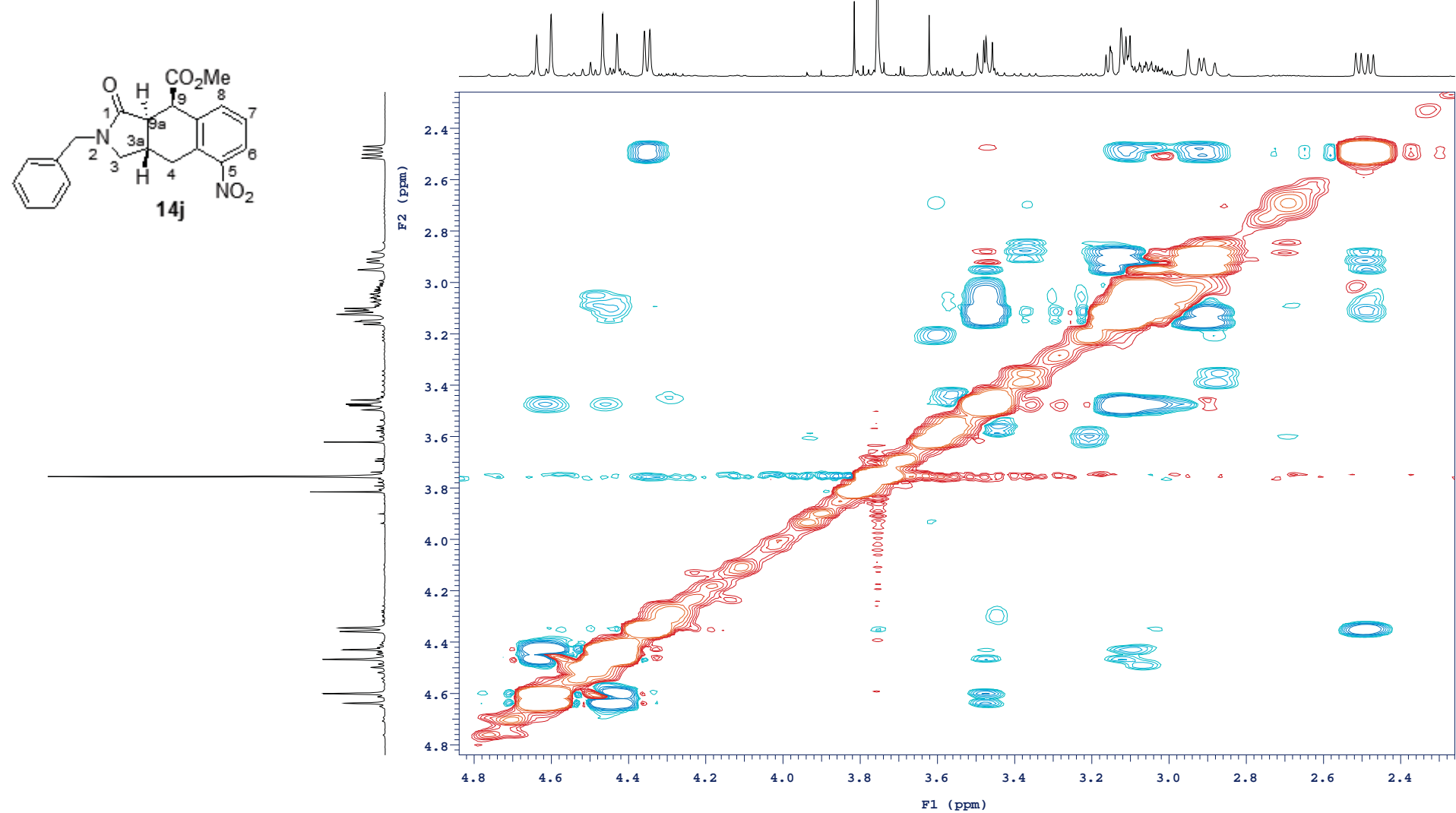

\section{Agilent Technologies}

\begin{tabular}{|c|c|c|c|}
\hline $\begin{array}{l}\text { Sample Name } \\
\text { Date collected 2015-11-16 }\end{array}$ & $\begin{array}{l}\text { Pulse sequence NOESY } \\
\text { Solvent cdcl3 }\end{array}$ & $\begin{array}{l}\text { Temperature }{ }^{3} \\
\text { Spectrometer }{ }_{\text {Agilent-NMR-inova } 400}\end{array}$ & $\begin{array}{l}\text { Study owner vnmr1 } \\
\text { Operator vnmr1 }\end{array}$ \\
\hline
\end{tabular}

$\operatorname{NOESY}\left(\mathrm{CDCl}_{3}, 400 \mathrm{MHz}\right)$

14k
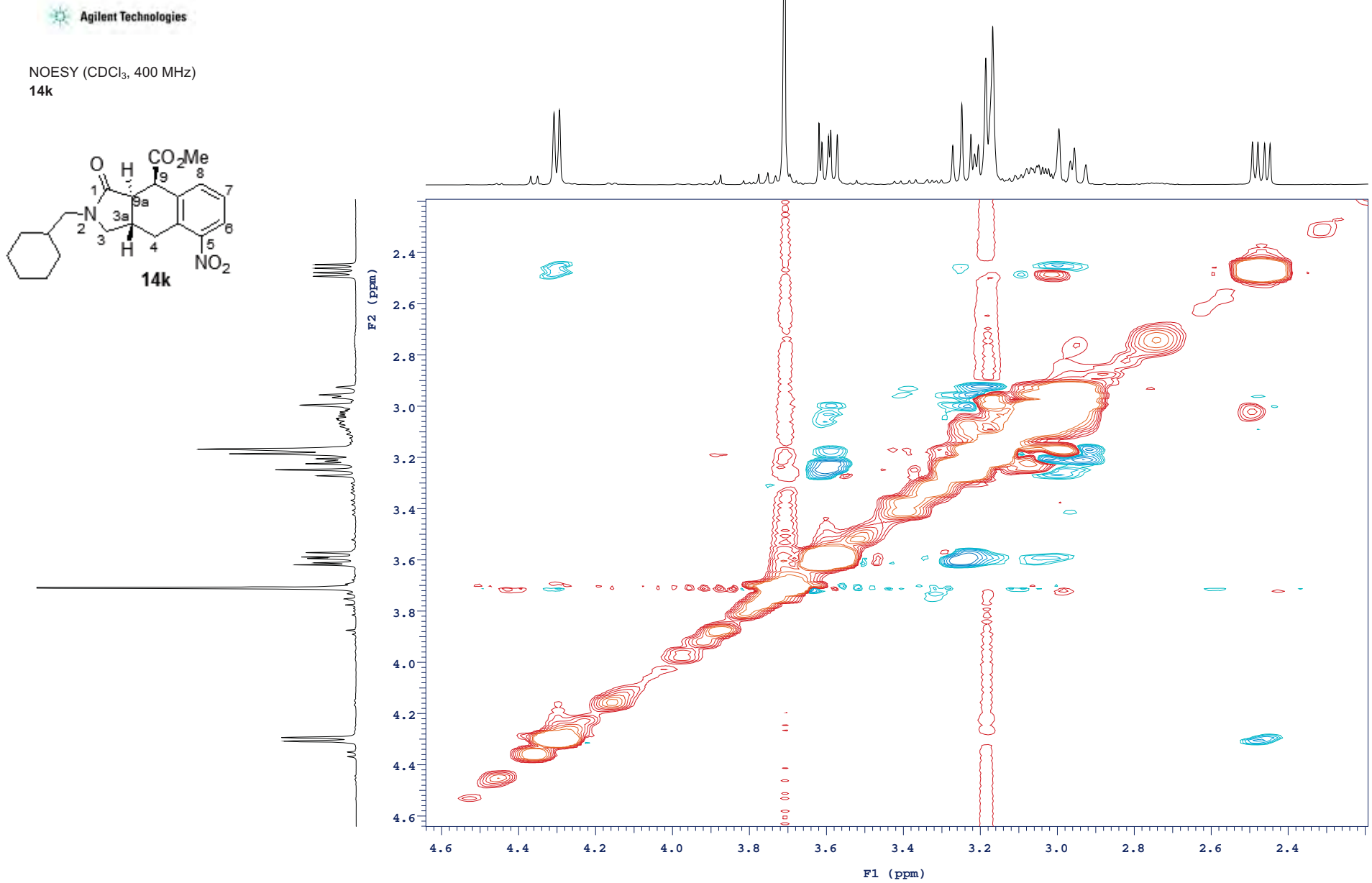
$\operatorname{NOESY}\left(\mathrm{CDCl}_{3}, 400 \mathrm{MHz}\right)$

15j
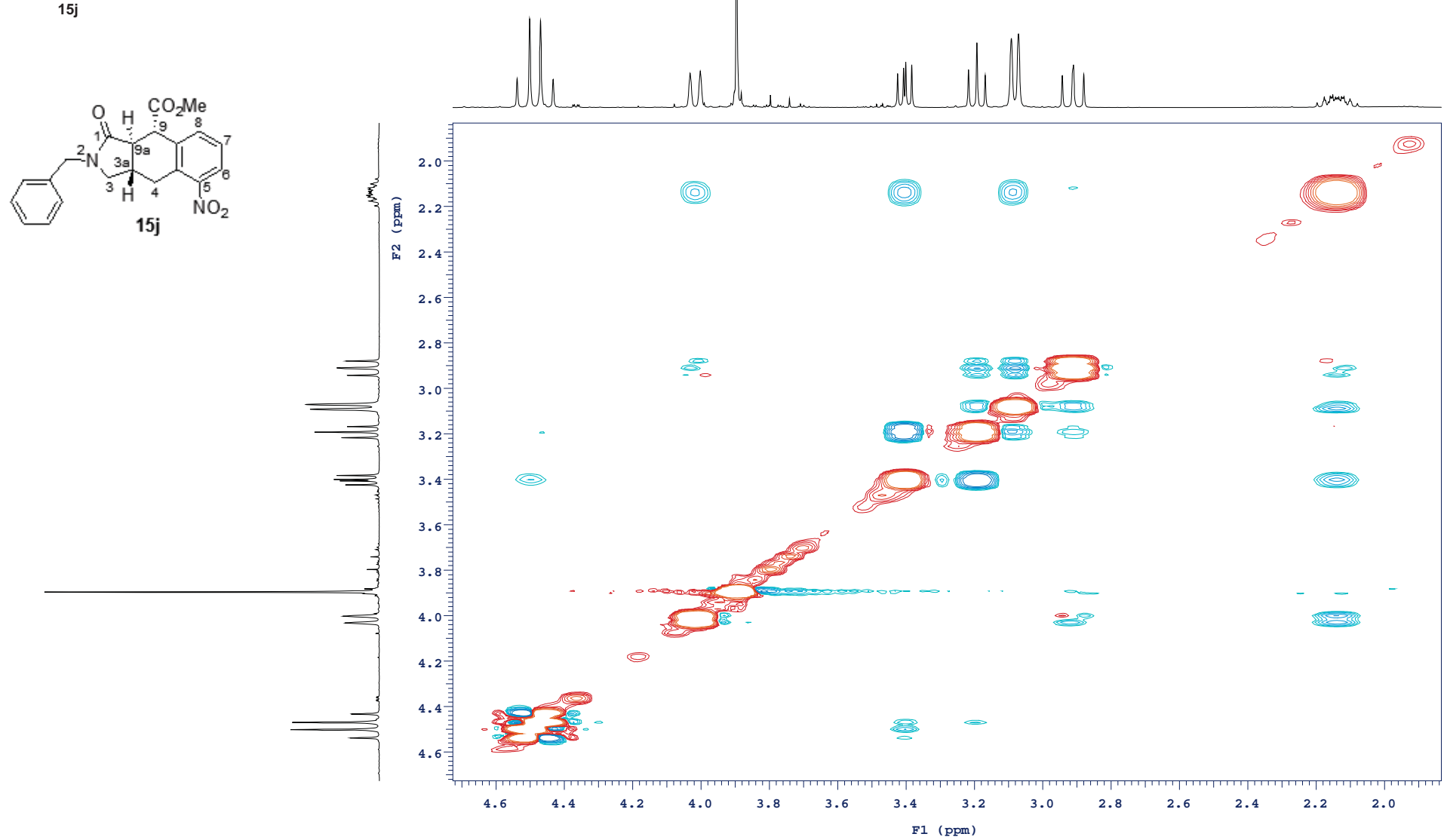

\section{क. Agilent Technologies}

\begin{tabular}{|c|c|c|}
\hline $\begin{array}{l}\text { Sample Na } \\
\text { Date coller }\end{array}$ & $\begin{array}{l}\text { Pulso sequence NOESY } \\
\text { Solvent cdcl3 }\end{array}$ & $\begin{array}{l}\text { Temperature }{ }^{3} \\
\text { Spectrometer Agilent-NMR-inova400 }\end{array}$ \\
\hline
\end{tabular}

NOESY $\left(\mathrm{CDCl}_{3}, 400 \mathrm{MHz}\right)$

$15 \mathrm{k}$

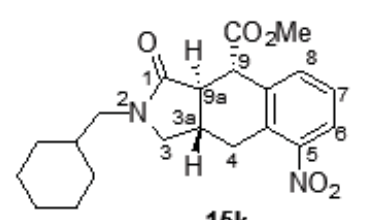

$15 k$

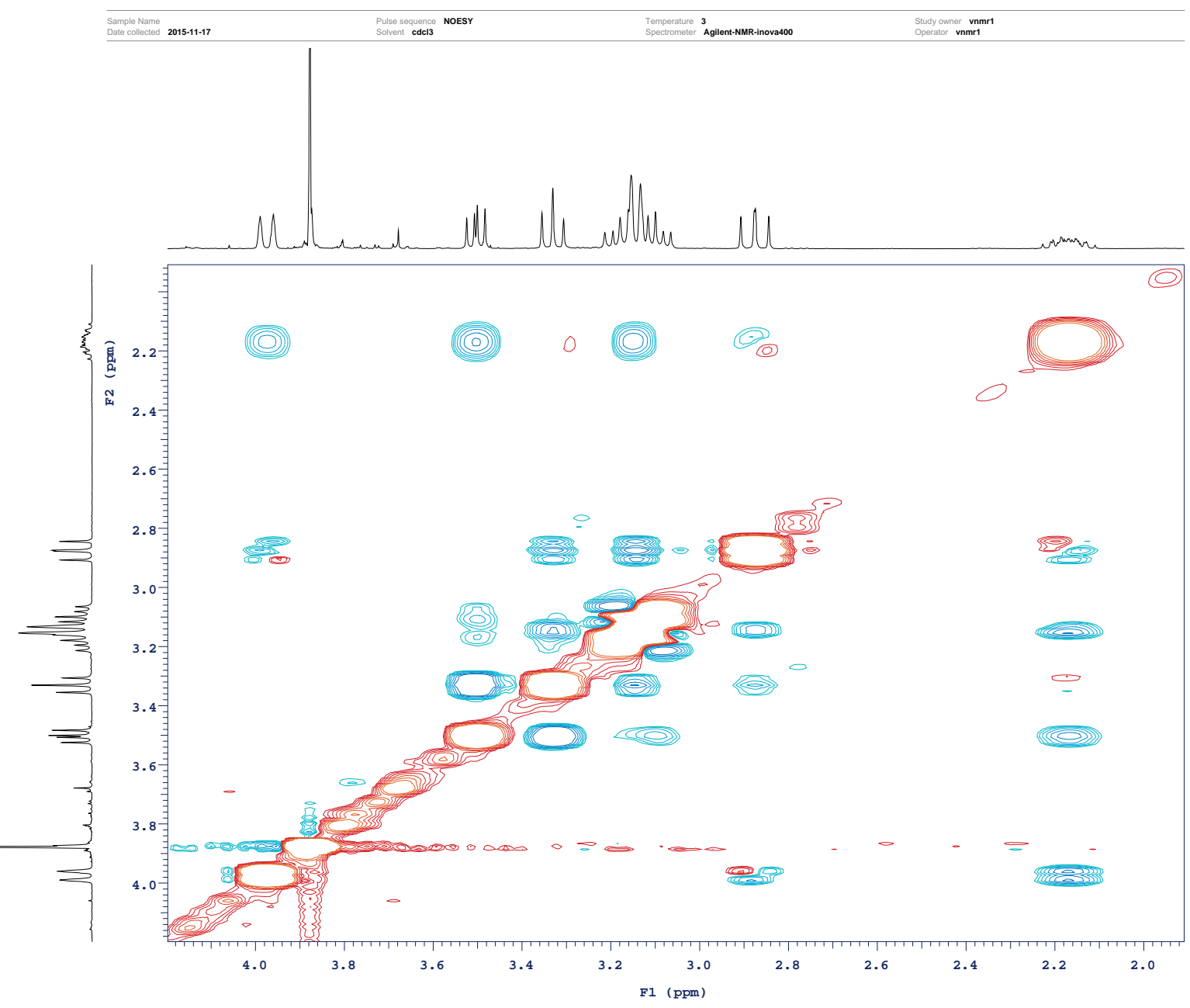

SSS21 

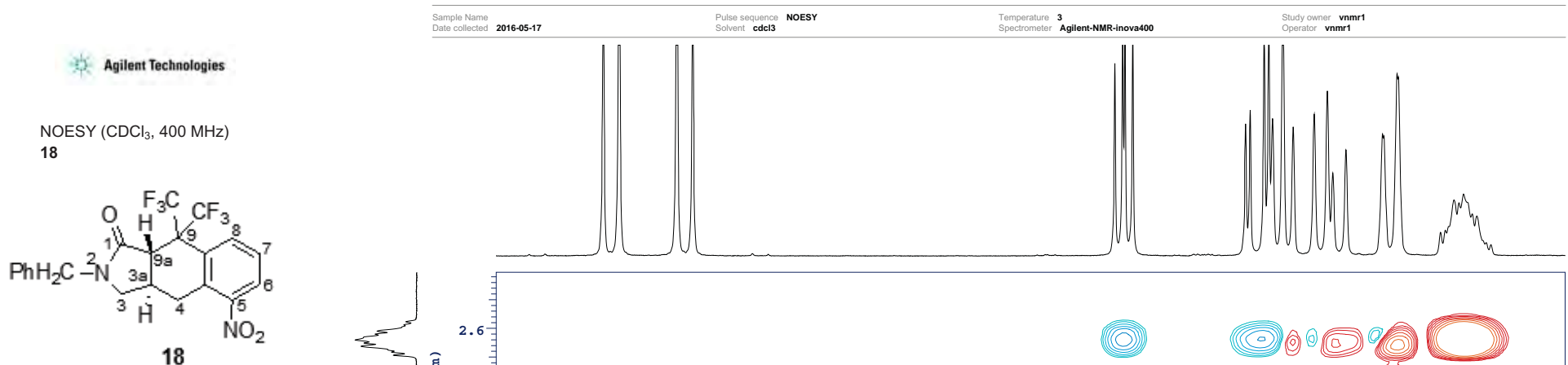

18

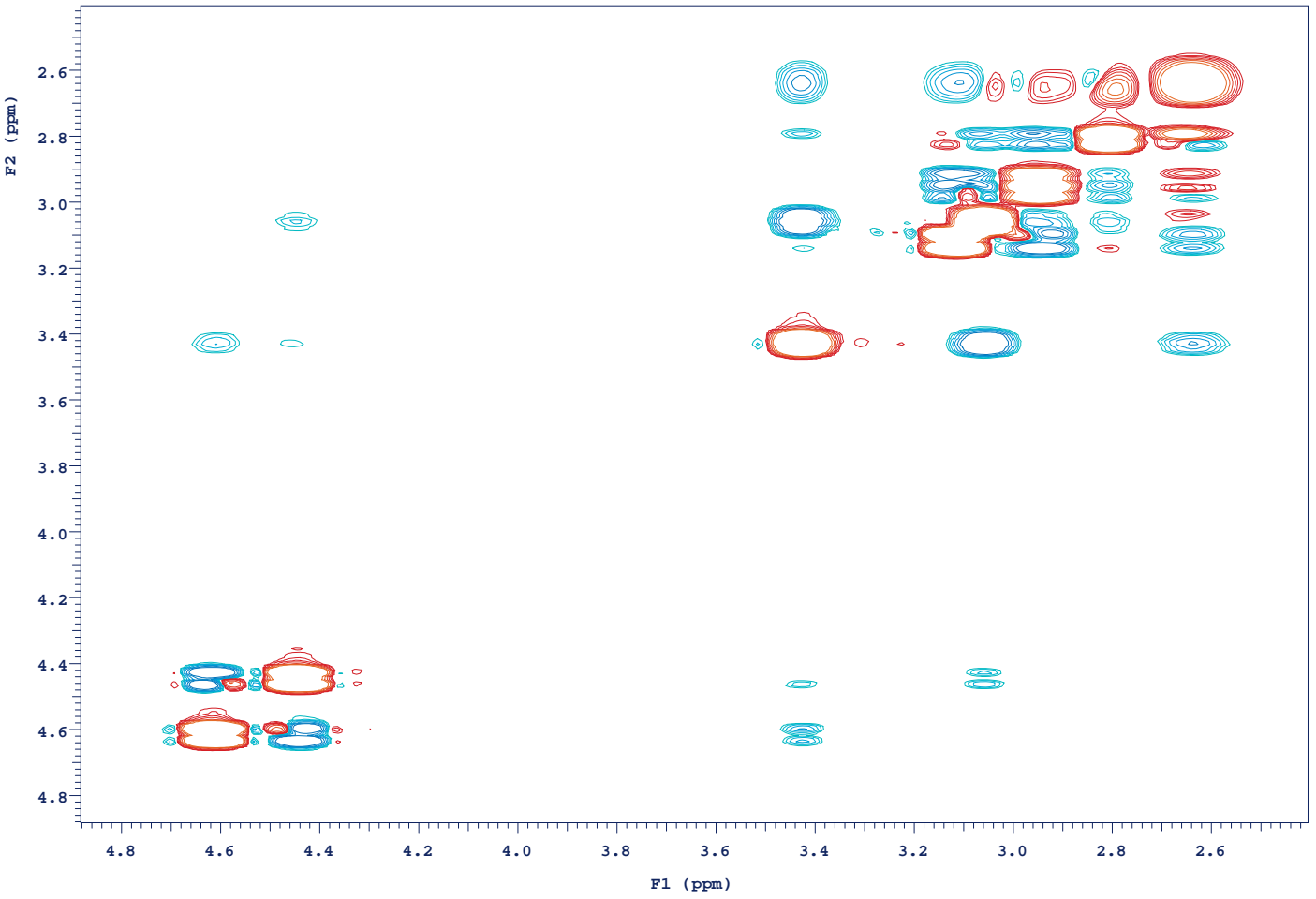

